TRANSACTIONS OF THE

AMERICAN MATHEMATICAL SOCIETY

Volume 362, Number 2, February 2010, Pages 957-983

S 0002-9947(09)04868-5

Article electronically published on September 10, 2009

\title{
ADDITION THEOREMS VIA CONTINUED FRACTIONS
}

\author{
MOURAD E. H. ISMAIL AND JIANG ZENG
}

\begin{abstract}
We show connections between a special type of addition formulas and a theorem of Stieltjes and Rogers. We use different techniques to derive the desirable addition formulas. We apply our approach to derive special addition theorems for Bessel functions and confluent hypergeometric functions. We also derive several addition theorems for basic hypergeometric functions. Applications to the evaluation of Hankel determinants are also given.
\end{abstract}

\section{INTRODUCTION}

An algebraic addition theorem for a function $f$ is an identity of the form

$$
P(f(x), f(y), f(x+y))=0
$$

for some polynomial $P$ in three variables. Weierstrass proved that an analytic function satisfying an algebraic addition theorem is a rational function in $z$, a rational function in $e^{\lambda z}$ for some $\lambda$, or an elliptic function, [11, Chapter 13]. This notion is too restricted to be useful in the theory of special functions. In general a family, say $\phi_{\lambda}$, of special functions satisfies an addition formula if there is an elementary continuous function $\Lambda$ of three variables $x, y, t$ and an expansion in terms of a family of special functions $\psi_{\mu}$ such that the expansion coefficients factor as products in $x$ and $y$. In other words, we have

$$
\phi_{\lambda}(\Lambda(x, y, t))=\sum_{\mu} C(\lambda, \mu) \phi_{\lambda}^{\mu}(x) \phi_{\lambda}^{\mu}(y) \psi_{\mu}(t), \quad C(\lambda, \mu) \in \mathbb{C} .
$$

Recall the definition of a Bessel function

$$
J_{\nu}(z)=\sum_{m=0}^{\infty} \frac{(-1)^{m}}{m ! \Gamma(m+\nu+1)}\left(\frac{x}{2}\right)^{2 m+\nu}=\frac{(z / 2)^{\nu}}{\Gamma(\nu+1)}{ }_{0} F_{1}\left(\begin{array}{c|c}
- \\
\nu+1
\end{array} \mid \frac{-z^{2}}{4}\right),
$$

and the modified Bessel function

$$
I_{\nu}(x):=e^{-i \nu \pi / 2} J_{\nu}\left(e^{i \pi / 2} x\right)=\sum_{m=0}^{\infty} \frac{(x / 2)^{\nu+2 m}}{m ! \Gamma(m+\nu+1)} .
$$

One important addition theorem is the addition theorem for Bessel functions,

$$
\frac{J_{\nu}(w)}{w^{\nu}}=\frac{\Gamma(\nu)}{(z Z / 2)^{\nu}} \sum_{n=0}^{\infty}(\nu+n) C_{n}^{\nu}(\cos \phi) J_{\nu+n}(z) J_{\nu+n}(Z),
$$

Received by the editors August 3, 2007 and, in revised form, May 5, 2008.

2000 Mathematics Subject Classification. Primary 33D15, 33C15; Secondary 30E05, 05A15.

Key words and phrases. Addition theorems, orthogonal polynomials, continued $J$-fractions, $q$-orthogonal polynomials, Askey-Wilson polynomials, Bessel and confluent hypergeometric functions.

(C)2009 American Mathematical Society Reverts to public domain 28 years from publication 
for $\nu \neq 0,-1,-2, \ldots$, where $w:=\left(z^{2}+Z^{2}-2 z Z \cos \phi\right)^{1 / 2}$, [7, (7.15.30)], [26]. The polynomials $\left\{C_{n}^{\nu}(x)\right\}$ are the ultraspherical polynomials. The special case $\phi=\pi$ is

$$
\frac{J_{\nu}(x+y)}{(x+y)^{\nu}}=\frac{\Gamma(\nu)}{(x y / 2)^{\nu}} \sum_{n=0}^{\infty}(\nu+n) \frac{(-1)^{n}(2 \nu)_{n}}{n !} J_{\nu+n}(x) J_{\nu+n}(y)
$$

since $C_{n}^{\nu}(-1)=(-1)^{n}(2 \nu)_{n} / n$ !. The addition theorems we will encounter in this work are of the type (1.6).

This work arose from an attempt to understand the Stieltjes-Rogers theorem of continued $J$-fractions; see Theorem 2.1. It is clear that (2.12) of Theorem 2.1 is an addition theorem of the type (1.6).

We decided to explore $q$-analogues of Rogers' addition formula (2.12) of Theorem 2.1 and to compute the functions $Q_{j}(x), j=0,1, \ldots$ for specific continued fractions, since the theory of orthogonal polynomials, especially the recently discovered one, provides a rich source of continued $J$-fractions. We discovered two $q$-analogues of Theorem 2.1. They are Theorems 4.1 and 4.4.

In this work we establish addition theorems for many special functions. To the best of our knowledge only (1.6) and (3.8) are known. We offer three different techniques of proof and provide at least one example of each technique as illustrations. We realize that it is possible to use fewer techniques to achieve the same goals, but we believe there is merit in utilizing as many different ideas as possible. One approach uses the plane wave expansion, [15, (4.8.2)],

$$
e^{x y}=\sum_{n=0}^{\infty} \frac{\Gamma(\alpha+\beta+n+1)}{\Gamma(\alpha+\beta+2 n+1)}(2 y)^{n} e^{-y}{ }_{1} F_{1}\left(\begin{array}{c}
\beta+n+1 \\
\alpha+\beta+2 n+2
\end{array} \mid 2 y\right) P_{n}^{(\alpha, \beta)}(x)
$$

for $\alpha>-1, \beta>-1$, and its special case [15, (4.8.3)],

$$
e^{x y}=\Gamma(\nu)(y / 2)^{-\nu} \sum_{n=0}^{\infty}(\nu+n) I_{\nu+n}(y) C_{n}^{\nu}(x), \quad \nu>-1 / 2 .
$$

The polynomials $\left\{P_{n}^{(\alpha, \beta}(x)\right\}$ and $\left\{C_{n}^{\nu}(x)\right\}$ are Jacobi and ultraspherical polynomials, respectively. The expansions (1.7)-(1.8) are instances of the Fields and Wimp expansions [9]; see also [8, 24]. Other techniques use Rodrigues-type formulas followed by integration by parts, and connection coefficient formulas.

We shall follow the notation and terminology in [1], [15], and [12]. In particular we shall use the Rogers connection coefficients formula for the continuous $q$-ultraspherical polynomials $\left\{C_{n}(x ; \beta \mid q)\right\}$,

$$
C_{n}(x ; \gamma \mid q)=\sum_{k=0}^{\lfloor n / 2\rfloor} \frac{\beta^{k}(\gamma / \beta)_{k}(\gamma)_{n-k}}{(q)_{k}(q \beta)_{n-k}} \frac{1-\beta q^{n-2 k}}{1-\beta} C_{n-2 k}(x ; \beta \mid q),
$$

[15, p. 330], and the facts that

$$
U_{n}(x)=C_{n}^{1}(x)=C_{n}(x ; q \mid q) .
$$

\section{Preliminaries}

Given a moment sequence $\left\{\mu_{n}\right\}$, we define the linear functional $\mathcal{L}: x^{n} \mapsto \mu_{n}$ on the vector space of polynomials $\mathbb{C}[x]$. We shall always assume $\mu_{0}=\mathcal{L}(1)=1$. Then the monic polynomials $P_{n}(x)$ orthogonal with respect to the $\mathcal{L}$ or the moment 
$\mu_{n}$ satisfy the following three-term recurrence relation (the spectral theorem for orthogonal polynomials [15, Chapter 2]):

$$
P_{n+1}(x)=\left(x-b_{n}\right) P_{n}(x)-\lambda_{n} P_{n-1}(x), \quad n \geq 0,
$$

where $\lambda_{0} P_{-1}(x)=0$ and $P_{0}(x)=1$. We shall always require the functional to be regular, 5], which is equivalent to demanding that $\lambda_{n} \neq 0$ for all $n, n>0$. The orthogonality relation is

$$
\mathcal{L}\left(P_{m} P_{n}\right)=\lambda_{1} \lambda_{2} \cdots \lambda_{n} \delta_{m, n} .
$$

The moment sequence is related to the coefficients $b_{n}$ and $\lambda_{n}$ by the following identity:

$$
1+\sum_{n \geq 1} \mu_{n} x^{n}=\frac{1}{1-b_{0} x-\frac{\lambda_{1} x^{2}}{1-b_{1} x-\frac{\lambda_{2} x^{2}}{\frac{\ddots}{1-b_{n} x-\frac{\lambda_{n} x^{2}}{\ddots}}}}} .
$$

Define the determinants

$$
\Delta_{i, n}=\left|\begin{array}{cccc}
\mu_{0} & \mu_{1} & \ldots & \mu_{i} \\
\mu_{1} & \mu_{2} & \ldots & \mu_{i+1} \\
\vdots & \vdots & \vdots & \vdots \\
\mu_{i-1} & \mu_{i} & \ldots & \mu_{2 i-1} \\
\mu_{n} & \mu_{n+1} & \ldots & \mu_{n+i}
\end{array}\right|, \quad D_{n}(x)=\left|\begin{array}{cccc}
\mu_{0} & \mu_{1} & \ldots & \mu_{n} \\
\mu_{1} & \mu_{2} & \ldots & \mu_{n+1} \\
\vdots & \vdots & \vdots & \vdots \\
\mu_{n-1} & \mu_{n} & \ldots & \mu_{2 n-1} \\
1 & x & \ldots & x^{n}
\end{array}\right| .
$$

In particular, let

$$
D_{n}=\Delta_{n, n}, \quad \chi_{n}=\Delta_{n, n+1} .
$$

Then $P_{n}(x)=\left(D_{n-1}\right)^{-1} D_{n}(x)$ is the monic orthogonal polynomial sequence for $\mathcal{L}$.

It is easy to see that

$$
\begin{aligned}
\mathcal{L}\left(x^{n} P_{n}(x)\right) & =\frac{D_{n}}{D_{n-1}}=\lambda_{n} \lambda_{n-1} \ldots \lambda_{1}, \\
\mathcal{L}\left(x^{n+1} P_{n}(x)\right) & =\frac{\chi_{n}}{D_{n-1}}=\lambda_{n} \lambda_{n-1} \ldots \lambda_{1}\left(b_{0}+\cdots+b_{n}\right) .
\end{aligned}
$$

Therefore

$$
\lambda_{n}=\frac{\left.\mathcal{L}\left[P_{n}^{2}(x)\right)\right]}{\left.\mathcal{L}\left[P_{n-1}^{2}(x)\right)\right]}=\frac{D_{n-2} D_{n}}{D_{n-1}^{2}}
$$

and

$$
b_{n}=\frac{\left.\mathcal{L}\left[x P_{n}^{2}(x)\right)\right]}{\left.\mathcal{L}\left[P_{n}^{2}(x)\right)\right]}=\frac{\chi_{n}}{D_{n}}-\frac{\chi_{n-1}}{D_{n-1}} .
$$

The next theorem is the backbone of this work.

Theorem 2.1. Define the Stieltjes tableau of entries $H_{i, n}(n \geq 0)$ by

$$
\begin{aligned}
H_{i, n} & =0 \quad \text { for } \quad i<0 \quad \text { and } \quad i>n ; \\
H_{n, n} & =1 \quad \text { for all } \quad n \geq 0 \\
H_{i, n} & =H_{i-1, n-1}+b_{i} H_{i, n-1}+\lambda_{i+1} H_{i+1, n-1} .
\end{aligned}
$$


Then the generating function $\sum_{n>0} H_{0, n} x^{n}$ has the continued fraction expansion (2.3) if and only if, for any two nonnegative integers $k, \ell \geq 0$, the following convolution identities

$$
H_{0, k+\ell}=H_{0, k} H_{0, \ell}+\lambda_{1} H_{1, k} H_{1, \ell}+\cdots+\lambda_{1} \cdots \lambda_{j} H_{j, k} H_{j, \ell}+\cdots
$$

hold. Moreover with the exponential generating functions of $\left\{Q_{j}(t)\right\}$,

$$
Q_{j}(t)=\sum_{n=j}^{\infty} H_{j, n} \frac{t^{n}}{n !}
$$

the convolution identity (2.10) is equivalent to the addition formula

$$
Q_{0}(x+y)=\sum_{n=0}^{\infty} \lambda_{1} \cdots \lambda_{n} Q_{n}(x) Q_{n}(y) .
$$

Wall 23 points out that the first part of Theorem 2.1 is due to Stieltjes, but the addition theorem (2.12) is due to Rogers. For a proof and references, see [23. Section 53].

It is important to note that the $H_{j, n}$ 's are the connection coefficients in

$$
x^{n}=\sum_{j=0}^{n} H_{j, n} P_{j}(x) .
$$

Observe that the addition formula (2.12) is equivalent to

$$
\frac{x h_{0}(x)-y h_{0}(y)}{x-y}=\sum_{n=0}^{\infty} \lambda_{1} \cdots \lambda_{n} h_{n}(x) h_{n}(y),
$$

where

$$
h_{j}(t)=\sum_{n=j}^{\infty} H_{j, n} t^{n} .
$$

Note that if $x=y$, then (2.14) reduces to

$$
x h_{0}^{\prime}(x)+h_{0}(x)=\sum_{n=0}^{\infty} \lambda_{1} \cdots \lambda_{n} h_{n}(x)^{2} .
$$

In general (2.10) implies

$$
\sum_{m=0}^{\infty} H_{0, m} \sum_{j=0}^{m} c_{j} s^{j} d_{m-j} t^{m-j}=\sum_{n=0}^{\infty} \lambda_{1} \cdots \lambda_{n} Q_{n}(s) R_{n}(t),
$$

where

$$
Q_{n}(s)=\sum_{j=n}^{\infty} H_{n, j} c_{j} s^{j}, \quad R_{n}(t)=\sum_{j=n}^{\infty} H_{n, j} d_{j} t^{j} .
$$

We may define a generalized translation operator $(G T)$ on polynomials by

$$
(G T)_{s} x^{m}=\sum_{j=0}^{m} c_{j} s^{j} d_{m-j} t^{m-j} .
$$

One can extend $(G T)_{s}$ to formal power series by linearity. 
Remark 2.2. According to the Flajolet-Viennot theory [10, 25] we can interpret $H_{i, n}$ in the Stieltjes' tableau as follows. Let us attach weights to the steps of a lattice path at level $i(i \geq 0)$ of a Motzkin path in the following way:

$$
w(/)=1, \quad w(-)=b_{i} \quad \text { and } \quad w(\backslash)=\lambda_{i} .
$$

Let $\Gamma_{0 \rightarrow i}(n)$ be the set of Motzkin paths from level 0 to level $i$ of length $n$. Then we have the following interpretation:

$$
\begin{aligned}
H_{i, n} & =\sum_{\gamma \in \Gamma_{0 \rightarrow i}(n)} w(\gamma), \\
\lambda_{1} \lambda_{2} \cdots \lambda_{i} H_{i, n} & =\sum_{\gamma \in \Gamma_{i \rightarrow 0}(n)} w(\gamma) .
\end{aligned}
$$

This provides a combinatorial interpretation of (2.10).

Let $\left\{P_{j}(x)\right\}$ be the monic orthogonal polynomials with respect to the moment sequence $H_{0, n}$ and $\mathcal{L}$ be the functional $\mathcal{L}\left(x^{n}\right)=H_{0, n}$. Then it follows from (2.13) that

$$
Q_{j}(t)=\sum_{n=j}^{\infty} \frac{\mathcal{L}\left(x^{n} P_{j}(x)\right)}{\mathcal{L}\left(P_{j}^{2}(x)\right)} \frac{t^{n}}{n !}=\frac{1}{\lambda_{1} \ldots \lambda_{j}} \mathcal{L}\left(P_{j}(x) e^{x t}\right) .
$$

Note that $b_{i}=H_{i+1, i+2}-H_{i, i+1}$ and

$$
H_{j, n}=\frac{\Delta_{j, n}}{\lambda_{1} \ldots \lambda_{j}} .
$$

Therefore the addition formula (2.12) generalizes the Hankel determinants in (2.4). We refer the readers to $3,13,14,20,21,28$ for the application of orthogonal polynomials to the computation of Hankel determinants.

The following theorem gives another interpretation of the function $Q_{0}(x)$ for which our technique will derive an addition theorem.

Theorem 2.3. Assume that $\left\{P_{n}(x)\right\}$ are orthogonal with respect to a positive measure $\mu$ with compact support contained in $\{z:|z|<r\}$. Then

$$
\lambda_{1} \lambda_{2} \ldots \lambda_{j} Q_{j}(t)=\oint_{|z|=r} e^{t / z} F_{n}(1 / z) \frac{d z}{z}
$$

where

$$
F_{n}(z):=\int_{\mathbb{R}} \frac{P_{n}(u)}{z-u} d \mu(u), \quad z \notin \operatorname{supp}\{\mu\} .
$$

Proof. Let $\mu_{n}=\int_{\mathbb{R}} x^{n} d \mu(x)$. The right-hand side of (2.20) is

$$
\begin{gathered}
\oint_{|z|=r} e^{t / z} \int_{\mathbb{R}} \frac{P_{j}(u)}{1-z u} d \mu(u) \frac{d z}{z}=\int_{\mathbb{R}} \oint_{|z|=r} \sum_{n=0}^{\infty} \frac{t^{n} z^{-n}}{n !} \sum_{k=0}^{\infty}(u z)^{k} P_{j}(u) d \mu(u) \frac{d z}{z} \\
=\int_{\mathbb{R}} \sum_{n=0}^{\infty} \frac{(t u)^{n}}{n !} P_{j}(u) d \mu(u)=\int_{\mathbb{R}} e^{t u} P_{j}(u) d \mu(u),
\end{gathered}
$$

and the theorem follows from (2.18). 
The function $F_{n}(z)$ is related to the function of the second kind [15].

Throughout this work we will use the Heine transformation

$$
{ }_{2} \phi_{1}(A, B, C, Z)=\frac{(B, A Z ; q)_{\infty}}{(C, Z ; q)_{\infty}}{ }_{2} \phi_{1}(C / B, Z ; A Z ; q, B)
$$

(see [12, (III.1)]) and the ${ }_{2} \phi_{1}$ to ${ }_{2} \phi_{2}$ transformation

$$
{ }_{2} \phi_{1}(A, B ; C ; q, Z)=\frac{(A Z ; q)_{\infty}}{(Z ; q)_{\infty}} \phi_{2}(A, C / B ; C, A Z ; q, B Z) .
$$

See [12, (III.4)].

\section{UltrasphericAl AND JACOBI POLYNOMIALS}

The ultraspherical (or Gegenbauer) polynomials are

$$
C_{n}^{\nu}(x)=\frac{(2 \nu)_{n}}{n !} F_{1}\left(\begin{array}{c|c}
-n, & n+2 \nu \\
\nu+1 / 2 & \frac{1-x}{2}
\end{array}\right), \quad \nu \neq 0 .
$$

The normalized weight function is

$$
w(x)=\left(1-x^{2}\right)^{\nu-1 / 2} A(\nu), \quad A(\nu)=\frac{\Gamma(\nu+1)}{\Gamma(1 / 2) \Gamma(\nu+1 / 2)} .
$$

The corresponding orthogonality functional $\mathcal{L}$ is defined by

$$
\mathcal{L}(f)=\int_{-1}^{1} f(x) w(x) d x
$$

The monic ultraspherical polynomials $\left\{P_{n}(x)\right\}$ and the $\lambda_{j}$ are

$$
P_{n}(x)=\frac{n !}{(\nu)_{n}} 2^{-n} C_{n}^{\nu}(x), \quad \lambda_{j}=\frac{j(j+2 \nu-1)}{4(\nu+j-1)(\nu+j)} .
$$

Moreover

$$
x P_{n}(x)=P_{n+1}(x)+\frac{n(n+2 \nu-1)}{4(\nu+n-1)(\nu+n)} P_{n-1}(x) .
$$

Therefore (2.18) when $\nu>-1 / 2$ implies

$$
\begin{aligned}
Q_{i}(x) & =\frac{1}{\lambda_{1} \lambda_{2} \ldots \lambda_{i}} \mathcal{L}\left(e^{x t} P_{i}(x)\right) \\
& =\frac{1}{\lambda_{1} \lambda_{2} \ldots \lambda_{i}} \Gamma(\nu)(t / 2)^{-\nu} \mathcal{L}\left(\sum_{n=0}^{\infty}(\nu+n) I_{\nu+n}(t) C_{n}^{\nu}(x) P_{i}(x)\right) \\
& =\Gamma(\nu)(t / 2)^{-\nu} \frac{2^{i}(\nu)_{i}}{i !}(\nu+i) I_{\nu+i}(t) .
\end{aligned}
$$

Therefore

$$
Q_{i}(t)=\frac{2^{i} \Gamma(\nu+i+1)}{i !(t / 2)^{\nu}} I_{\nu+i}(t) .
$$

It is straightforward to see that addition formula (2.12) in the present example is equivalent to (1.6).

Next we consider Jacobi polynomials. The normalized weight function is

$$
w(x)=(1-x)^{\alpha}(1+x)^{\beta} A(\alpha, \beta), \quad A(\alpha, \beta)=\frac{\Gamma(\alpha+\beta+2)}{2^{\alpha+\beta+1} \Gamma(\alpha+1) \Gamma(\beta+1)} .
$$


In this case, the functional $\mathcal{L}$ to be considered is

$$
\mathcal{L}(f)=\int_{-1}^{1} f(x) w(x) d x
$$

The monic Jacobi polynomials $\left\{P_{n}(x)\right\}$ are defined through

$$
P_{n}^{(\alpha, \beta)}(x)=\frac{(n+\alpha+\beta+1)_{n}}{2^{n} n !} P_{n}(x),
$$

so that

$$
\lambda_{n}=\frac{4 n(n+\alpha)(n+\beta)(n+\alpha+\beta)}{(2 n+\alpha+\beta-1)(2 n+\alpha+\beta)^{2}(2 n+\alpha+\beta+1)} .
$$

As in the case of ultraspherical polynomials we can use (1.7) and the result is

$$
Q_{i}(x)=\frac{t^{i} e^{-t}}{i !}{ }_{1} F_{1}\left(\begin{array}{c|c}
\beta+i+1 \\
\alpha+\beta+2 i+2
\end{array} \mid 2 t\right) .
$$

As an example of the use of the Rodrigues formulas we give another derivation of (3.4). The Rodrigues formula for Jacobi polynomials is [15, (4.2.8)]

$$
(1-x)^{\alpha}(1+x)^{\beta} P_{n}^{(\alpha, \beta)}(x)=\frac{(-1)^{n}}{2^{n} n !} \frac{d^{n}}{d x^{n}}\left[(1-x)^{n+\alpha}(1+x)^{n+\beta}\right] .
$$

Therefore

$$
\begin{aligned}
Q_{j}(t) & =\frac{2^{j} j ! A(\alpha, \beta)}{\lambda_{1} \ldots \lambda_{j}(\alpha+\beta+j+1)_{j}} \int_{-1}^{1} e^{x t}(1-x)^{\alpha}(1+x)^{\beta} P_{j}^{(\alpha, \beta)}(x) d x \\
& =\frac{(-1)^{j} A(\alpha, \beta)}{\lambda_{1} \ldots \lambda_{j}(\alpha+\beta+j+1)_{j}} \int_{-1}^{1} e^{x t} \frac{d^{j}}{d x^{j}}\left[(1-x)^{j+\alpha}(1+x)^{j+\beta}\right] d x \\
& =\frac{t^{j} A(\alpha, \beta)}{\lambda_{1} \ldots \lambda_{j}(\alpha+\beta+j+1)_{j}} \int_{-1}^{1} e^{x t}(1-x)^{j+\alpha}(1+x)^{j+\beta} d x,
\end{aligned}
$$

after integration by parts. Taking into account the integral representation [6, 6.5.1)]

$$
{ }_{1} F_{1}(a ; c ; z)=\frac{\Gamma(c)}{\Gamma(a) \Gamma(c-a)} \int_{0}^{1} e^{z u} u^{a-1}(1-u)^{c-a-1} d u, \quad \operatorname{Re} c>\operatorname{Re} a>0,
$$

(3.3), and (3.2), we see that the last expression for $Q_{j}(x)$ reduces to

$$
Q_{j}(t)=\frac{t^{j}}{j !} e^{t}{ }_{1} F_{1}\left(\begin{array}{c}
\alpha+j+1 \\
\alpha+\beta+2 j+2
\end{array} \mid-2 t\right) .
$$

The equivalence of the representations (3.4) and (3.7) follows from the transformation [15, (1.4.11)]

$$
{ }_{1} F_{1}\left(\begin{array}{c|c}
a & z \\
c & z
\end{array}\right)=e^{z}{ }_{1} F_{1}\left(\begin{array}{c|c}
c-a & -z) . \\
c &
\end{array}\right.
$$

This analysis establishes the following theorem, which is the main result of this section. 
Theorem 3.1. We have the addition theorem for the confluent hypergeometric functions

$$
\begin{aligned}
{ }_{1} F_{1}\left(\begin{array}{c}
\alpha+1 \\
\alpha+\beta+2
\end{array} \mid t+s\right)=\sum_{n=0}^{\infty} \frac{(\alpha+1)_{n}(\beta+1)_{n}(\alpha+\beta+1)_{n}}{(\alpha+\beta+1)_{2 n}(\alpha+\beta+2)_{2 n}} \frac{(t s)^{n}}{n !} \\
\quad \times{ }_{1} F_{1}\left(\begin{array}{c}
\alpha+n+1 \\
\alpha+\beta+2 n+2
\end{array} \mid t\right){ }_{1} F_{1}\left(\begin{array}{c}
\alpha+n+1 \\
\alpha+\beta+2 n+2
\end{array} \mid s\right) .
\end{aligned}
$$

When $\alpha=\beta=\nu-1 / 2$, Theorem 3.1 reduces to (1.6) since

$$
e^{-x}{ }_{1} F_{1}(\nu+1 / 2 ; 2 \nu+1 ; 2 x)=\Gamma(\nu+1)(2 / x)^{\nu} I_{\nu}(x),
$$

[6. (6.9.10)]. Moreover both (3.4) and (3.7) also reduce to (3.1).

Note that Theorem 3.1 and (3.1) can be proved from Theorem 2.3 and the facts

$$
\begin{gathered}
\int_{-1}^{1}(1-t)^{\alpha}(1+t)^{\beta} \frac{P_{n}^{(\alpha, \beta)}(t)}{z-t} d t=\frac{\Gamma(n+\alpha+1) \Gamma(n+\beta+1)}{\Gamma(2 n+\alpha+\beta+2)} \\
\times \frac{2^{n+\alpha+\beta+1}}{(z-1)^{n+1}}{ }_{2} F_{1}\left(\begin{array}{c}
n+1, \alpha+n+1 \\
\alpha+\beta+2 n+2
\end{array} \mid \frac{2}{1-z}\right)
\end{gathered}
$$

see (4.4.1) and (4.4.6) in [15]. Moreover the integral

$$
\int_{-1}^{1} e^{x t}(1-x)^{\alpha}(1+x)^{\beta} P_{n}^{(\alpha, \beta)}(x) d x
$$

can be evaluated from the plane wave expansion (1.7) instead of the use of Rodrigues' formula.

It must be noted that (3.8) coincides with formula (42) of Burchnall and Chaundy [4. It is also a limiting case of formula (50) 4]. The latter is stated in [6]; see the first unnumbered formula after (7) in Section 2.5.2. Indeed if we replace $z$ by $z / b$ and $\zeta$ by $\zeta / b$ and let $b \rightarrow \infty$, the above-mentioned formula reduces to our (3.8). The terminating case $\alpha=-m-1, \beta=\gamma+m$ of (3.8) is the case $r=0$ of Koornwinder's addition formula for Laguerre polynomials; see (3.3) in [19. Also, this terminating case of (3.8) is the inverse of 10.12(42) in [7].

\section{TWO $q$-ADDITION FORMULAS}

The $q$-binomial formula

$$
\sum_{n=0}^{\infty} \frac{(a ; q)_{n}}{(q ; q)_{n}} t^{n}=\frac{(a t ; q)_{\infty}}{(t ; q)_{\infty}}
$$

yields Euler's $q$-analogues of the exponential formulas:

$$
\begin{aligned}
& \sum_{n=0}^{\infty} \frac{t^{n}}{(q ; q)_{n}}=\frac{1}{(t ; q)_{\infty}}, \\
& \sum_{n=0}^{\infty} \frac{q^{\left(\begin{array}{c}
n \\
2
\end{array}\right)} t^{n}}{(q ; q)_{n}}=(-t ; q)_{\infty} .
\end{aligned}
$$

The $q$-difference operator is defined by

$$
\mathcal{D}_{q} f(z)=\frac{f(z)-f(q z)}{(1-q) z} .
$$


It is easy to see that

$$
\begin{aligned}
\mathcal{D}_{q}^{j}\left((-x t ; q)_{\infty}\right) & =\frac{t^{j} q^{\left(\frac{3}{2}\right)}}{(1-q)^{j}}\left(-x t q^{j} ; q\right)_{\infty}, \\
\mathcal{D}_{q}^{j}\left(\frac{1}{(x t ; q)_{\infty}}\right) & =\frac{t^{j}(1-q)^{-j}}{(x t ; q)_{\infty}} .
\end{aligned}
$$

Note also that

$$
\begin{aligned}
& \mathcal{D}_{1 / q}^{j}\left(\frac{1}{(x t ; q)_{\infty}}\right)=\frac{t^{j} q^{-\left(\begin{array}{c}
j \\
2
\end{array}\right)}}{(1-q)^{j}} \frac{1}{\left(x t q^{-j} ; q\right)_{\infty}} \\
& \mathcal{D}_{1 / q}^{j}\left((-x t ; q)_{\infty}\right)=\frac{t^{j}}{(1-q)^{j}}(-x t ; q)_{\infty} .
\end{aligned}
$$

4.1. First $q$-addition formula. Define the $q$-translation operator $\mathcal{T}_{s, q}$ by

$$
\mathcal{T}_{y, q} x^{n}=(x+y)(x+y q) \cdots\left(x+y q^{n-1}\right) .
$$

Extend this by linearity on functions $f(x)=\sum_{n=0}^{\infty} a_{n} x^{n}$, so that

$$
\mathcal{T}_{y, q} f(x)=\sum_{n=0}^{\infty} a_{n}(x+y)(x+y q) \cdots\left(x+y q^{n-1}\right) .
$$

Note that

$$
\mathcal{T}_{y, q^{-1}} x^{n}=(x+y)(x+y / q) \cdots\left(x+y / q^{n-1}\right)=\mathcal{T}_{x, q} y^{n} q^{-\left(\begin{array}{c}
n \\
2
\end{array}\right)} .
$$

Define two $q$-analogues of $Q_{j}(t)$ by

$$
Q_{j}(t ; q)=\sum_{n=j}^{\infty} H_{j, n} \frac{t^{n}}{(q ; q)_{n}}, \quad \widetilde{Q}_{j}(t ; q)=\sum_{n=j}^{\infty} H_{j, n} \frac{q^{\left(\begin{array}{c}
n \\
2
\end{array}\right)} t^{n}}{(q ; q)_{n}} .
$$

Theorem 4.1. The convolution identity (2.10) is equivalent to the addition formula

$$
\mathcal{T}_{s, q} Q_{0}(t ; q)=\sum_{n=0}^{\infty} \lambda_{1} \cdots \lambda_{n} Q_{n}(t ; q) \widetilde{Q}_{n}(s ; q) .
$$

Moreover, we have

$$
\begin{aligned}
& Q_{j}(t ; q)=\frac{1}{\lambda_{1} \cdots \lambda_{j}} \mathcal{L}\left(\frac{P_{j}(x)}{(x t ; q)_{\infty}}\right), \\
& \widetilde{Q}_{j}(t ; q)=\frac{1}{\lambda_{1} \cdots \lambda_{j}} \mathcal{L}\left(P_{j}(x)(-x t ; q)_{\infty}\right) .
\end{aligned}
$$

Proof. The $q$-binomial theorem and the definition of $\mathcal{T}_{y}$ give

$$
\mathcal{T}_{y, q} x^{n}=(x+y)(x+y q) \cdots\left(x+y q^{n-1}\right)=\sum_{k=0}^{n}\left[\begin{array}{l}
n \\
k
\end{array}\right]_{q} q^{\left(\begin{array}{c}
k \\
2
\end{array}\right)} x^{n-k} y^{k} .
$$

This establishes the equivalence of (4.11) and (2.10). Equations (4.12) and (4.13) follow from Euler's formulas (4.2)-(4.3). 
4.2. Little Jacobi polynomials. The little $q$-Jacobi polynomials $\left\{p_{n}(x ; a, b)\right\}$ are defined by

$$
p_{n}(x ; a, b)={ }_{2} \phi_{1}\left(q^{-n}, a b q^{n+1} ; a q ; q, q x\right) .
$$

See [18, pp. 92-93]. The corresponding monic polynomials $\left\{P_{j}(x)\right\}$ are given by

$$
p_{n}(x ; a, b)=\frac{(-1)^{n} q^{-\left(\begin{array}{c}
n \\
2
\end{array}\right)\left(a b q^{n+1} ; q\right)_{n}}}{(a q ; q)_{n}} P_{n}(x)
$$

and

$$
\lambda_{n}=\frac{a q^{2 n-1}\left(1-q^{n}\right)\left(1-a q^{n}\right)\left(1-b q^{n}\right)\left(1-a b q^{n}\right)}{\left(1-a b q^{2 n-1}\right)\left(1-a b q^{2 n}\right)\left(1-a b q^{2 n}\right)\left(1-a b q^{2 n+1}\right)} .
$$

Let

$$
a=q^{\alpha}, \quad b=q^{\beta} .
$$

Then the weight function is given by

$$
w(x ; \alpha, \beta)=\frac{(q x ; q)_{\infty}}{\left(q^{\beta+1} x ; q\right)_{\infty}} x^{\alpha},
$$

and the corresponding orthogonality functional is

$$
\mathcal{L}(f)=\frac{(a q, b q ; q)_{\infty}}{\left(a b q^{2}, q ; q\right)_{\infty}(1-q)} \int_{0}^{1} f(x) w(x ; \alpha, \beta) d_{q} x,
$$

where the $q$-integral is defined (see [12, p.23]) by

$$
\int_{0}^{a} f(t) d_{q} t:=a(1-q) \sum_{n=0}^{\infty} f\left(q^{n} a\right) q^{n}
$$

and

$$
\int_{a}^{b} f(t) d_{q} t=\int_{0}^{b} f(t) d_{q} t-\int_{0}^{a} f(t) d_{q} t
$$

The Rodrigues-type formula for the little $q$-Jacobi polynomials is

$$
p_{j}(x ; a, b)=\frac{1}{w(x ; \alpha, \beta)} \frac{(1-q)^{j} q^{j \alpha+\left(\begin{array}{l}
j \\
2
\end{array}\right)}}{\left(q^{\alpha+1} ; q\right)_{j}} \mathcal{D}_{1 / q}^{j}(w(x ; \alpha+j, \beta+j)) .
$$

Therefore

$$
\begin{aligned}
& \widetilde{Q}_{j}(t ; q) \\
& \quad=\frac{a^{-j} q^{-j^{2}}\left(a b q, a b q^{2} ; q\right)_{2 j}(a q, b q ; q)_{\infty}}{(q, a q, b q, a b q ; q)_{j}\left(a b q^{2}, q ; q\right)_{\infty}(1-q)} \int_{0}^{1}(-x t ; q)_{\infty} w(x ; \alpha, \beta) P_{j}(x) d_{q} x \\
& \quad=\frac{a^{-j} q^{-j^{2}}\left(a b q^{2} ; q\right)_{2 j}(a q, b q ; q)_{\infty}}{(q, b q ; q)_{j}\left(a b q^{2}, q ; q\right)_{\infty}(1-q)}(-1)^{j} q^{\left(\frac{j}{2}\right)} \int_{0}^{1}(-x t ; q)_{\infty} w(x ; \alpha, \beta) p_{j}(x ; a, b) d_{q} x \\
& \quad=\frac{q^{-j}\left(a b q^{2} ; q\right)_{2 j}(a q, b q ; q)_{\infty}(-1)^{j}(1-q)^{j}}{(q, a q, b q ; q)_{j}\left(a b q^{2}, q ; q\right)_{\infty}(1-q)} \int_{0}^{1}(-x t ; q)_{\infty} D_{1 / q}^{j}(w(x ; \alpha+j, \beta+j)) d_{q} x .
\end{aligned}
$$

The $q$-analogue of integration by parts is

$$
\int_{a}^{b} \mathcal{D}_{q}(f(x)) w(x) d_{q} x=-\frac{1}{q} \int_{a}^{b} f(x) \mathcal{D}_{1 / q}(w(x)) d_{q} x,
$$


provided that $w(a / q)=w(b / q)=0$; see [15, (11.4.9)]. Applying (4.20) to the last expression for $\widetilde{Q}_{j}(t ; q)$ we obtain

$$
\begin{aligned}
& \widetilde{Q}_{j}(t ; q)=\frac{\left(a b q^{2} ; q\right)_{2 j}(a q, b q ; q)_{\infty} t^{j} q^{\left(\begin{array}{l}
j \\
2
\end{array}\right)}}{(q, a q, b q ; q)_{j}\left(a b q^{2}, q ; q\right)_{\infty}} \sum_{n=0}^{\infty} \frac{\left(-t q^{j+n}, q^{n+1} ; q\right)_{\infty}}{\left(q^{\beta+n+1+j} ; q\right)_{\infty}} q^{(\alpha+j+1) n} \\
& =\frac{\left(-t q^{j}, a q^{j+1} ; q\right)_{\infty} t^{j} q^{\left(\frac{j}{2}\right)}}{(q ; q)_{j}\left(a b q^{2+2 j} ; q\right)_{\infty}}{ }_{2} \phi_{1}\left(\begin{array}{c|c}
b q^{j+1}, & 0 \\
-t q^{j} & q, a q^{j+1}
\end{array}\right) .
\end{aligned}
$$

This shows that

$$
\widetilde{Q}_{j}(t ; q)=\frac{t^{j} q^{\left(\begin{array}{c}
j \\
2
\end{array}\right)}}{(q ; q)_{j}} \lim _{\epsilon \rightarrow 0} \frac{\left(-t q^{j}, a q^{j+1} ; q\right)_{\infty}}{\left(a b q^{2+2 j}, \epsilon ; q\right)_{\infty}}{ }_{2} \phi_{1}\left(\begin{array}{c}
b q^{j+1}, \epsilon \\
-t q^{j}
\end{array} \mid q, a q^{j+1}\right) .
$$

Now the Heine transformation (2.22) leads to

$$
\frac{\left(-t q^{j}, a q^{j+1} ; q\right)_{\infty}}{\left(a b q^{2+2 j}, \epsilon ; q\right)_{\infty}}{ }_{2} \phi_{1}\left(\begin{array}{c}
b q^{j+1}, \epsilon \\
-t q^{j}
\end{array} \mid q, a q^{j+1}\right)={ }_{2} \phi_{1}\left(\begin{array}{c}
-t q^{j} / \epsilon, a q^{j+1} \\
a b q^{2 j+2}
\end{array} \mid q, \epsilon\right) .
$$

Therefore the above equation and (4.21) establish the basic hypergeometric representation

$$
\widetilde{Q}_{j}(t ; q)=\frac{t^{j} q^{\left(\begin{array}{c}
j \\
2
\end{array}\right)}}{(q ; q)_{j}}{ }_{1} \phi_{1}\left(\begin{array}{c}
a q^{j+1} \\
a b q^{2 j+2}
\end{array} \mid q,-t q^{j}\right) .
$$

Similarly we have

$$
\begin{aligned}
Q_{j}(t ; q) & =\frac{\left(a b q^{2} ; q\right)_{2 j}(a q, b q ; q)_{\infty} t^{j}}{(q, a q, b q ; q)_{j}\left(a b q^{2}, q ; q\right)_{\infty}} \sum_{n=0}^{\infty} \frac{\left(q^{1+n} ; q\right)_{\infty} a^{n} q^{(j+1) n}}{\left(b q^{n+1+j}, t q^{n} ; q\right)_{\infty}} \\
& =\frac{t^{j}\left(a q^{j+1} ; q\right)_{\infty}}{(q ; q)_{j}\left(t, a b q^{2 j+2} ; q\right)_{\infty}}{ }_{2} \phi_{1}\left(\begin{array}{cc|c}
b q^{j+1}, & t & q, a q^{j+1} \\
0 &
\end{array}\right) .
\end{aligned}
$$

Applying the Heine transformation (2.22) to the last ${ }_{2} \phi_{1}$ yields

$$
Q_{j}(t ; q)=\frac{t^{j}}{(q ; q)_{j}}{ }^{2} \phi_{1}\left(\begin{array}{c|c}
0, a q^{j+1} \\
a b q^{2 j+2}
\end{array} \mid q, t\right) .
$$

Theorem 4.2. For the functional in (4.18) the functions $\widetilde{Q}_{j}(t ; q)$ and $Q_{j}(t ; q)$ are given by (4.22) and (4.23). Moreover we have the following addition formula:

$$
\begin{aligned}
& { }_{2} \phi_{1}\left(\begin{array}{c}
a q,-s / t \\
a b q^{2}
\end{array} \mid q, t\right)=\sum_{j=0}^{\infty} \frac{q^{3\left(\begin{array}{c}
j \\
2
\end{array}\right)(a q, b q, a b q ; q)_{j}}}{(q ; q))_{j}\left(a b q, a b q^{2} ; q\right)_{2 j}}(q a s t)^{j} \\
& \times{ }_{2} \phi_{1}\left(\begin{array}{c|c}
0, a q^{j+1} \\
a b q^{2 j+2}
\end{array} \mid q, t\right){ }_{1} \phi_{1}\left(\begin{array}{c}
a q^{j+1} \\
a b q^{2 j+2}
\end{array} \mid q,-s q^{j}\right) .
\end{aligned}
$$

Proof. We only need to show that the left-hand side of (4.24) is $\mathcal{T}_{s, q} Q_{0}(t, q)$. This is indeed the case as can be seen from [4.8).

It is important to note that Theorem 4.2 is a $q$-analogue of Theorem 3.1. Indeed with $a=q^{\alpha}, b=q^{\beta}$ and $s$ and $t$ replaced by $s(1-q)$ and $t(1-q)$, respectively, equation (4.24) tends to (3.8) as $q \rightarrow 1^{-}$.

Note that the transformation (2.23) implies

$$
Q_{j}(t ; q)=\frac{t^{j}}{(q ; q)_{j}(t ; q)_{\infty}}{ }_{1} \phi_{1}\left(\begin{array}{c}
b q^{j+1} \\
a b q^{2 j+2}
\end{array} \mid q, q^{j+1} a t\right) .
$$


Thus the addition theorem (4.24) has the alternate form

$$
\begin{aligned}
& { }_{2} \phi_{1}\left(\begin{array}{c|c}
a q, \\
a b q^{2}
\end{array} \mid q, t\right)=\sum_{j=0}^{\infty} \frac{q^{3\left(\begin{array}{c}
j \\
2
\end{array}\right)(a q, b q, a b q ; q)_{j}}}{(q ; q)_{j}\left(a b q, a b q^{2} ; q\right)_{2 j}} \frac{(q a s t)^{j}}{(t ; q)_{\infty}} \\
& \quad \times{ }_{1} \phi_{1}\left(\begin{array}{c}
b q^{j+1} \\
a b q^{2 j+2}
\end{array} \mid q, q^{j+1} a t\right){ }_{1} \phi_{1}\left(\begin{array}{c}
a q^{j+1} \\
a b q^{2 j+2}
\end{array} \mid q,-q^{j} s\right) .
\end{aligned}
$$

Remark. A different $q$-analogue of Theorem 3.1 was given by Jackson [17, (55)].

4.3. Big $q$-Jacobi polynomials. The monic big $q$-Jacobi polynomials are

$$
p_{n}(x ; a, b, c)=\frac{\left(a b q^{n+1} ; q\right)_{n}}{(a q, c q ; q)_{n}} P_{n}(x) .
$$

Let

$$
w_{1}(x, a, b, c)=\frac{(x / a, x / c)_{\infty}}{(x, b x / c)_{\infty}}
$$

and

$$
w(x, a, b, c)=\frac{w_{1}(x, a, b, c)}{a q(1-q)} \frac{(a q, b q, c q, a b q / c ; q)_{\infty}}{\left(q, a b q^{2}, c / a, a q / c ; q\right)_{\infty}} .
$$

The corresponding orthogonality functional is

$$
\mathcal{L}(f)=\int_{c q}^{a q} f(x) w(x, a, b, c) d_{q} x .
$$

The Rodrigues-type formula for big $q$-Jacobi polynomials is

$$
w_{1}(x) p_{n}(x ; a, b, c)=\frac{a^{n} c^{n} q^{n(n+1)}(1-q)^{n}}{(a q, c q ; q)_{n}} \mathcal{D}_{q}^{n} w_{1}\left(x, a q^{n}, b q^{n}, c q^{n}\right) .
$$

Note that

$$
\lambda_{n}=\frac{-a c q^{n+1}\left(1-q^{n}\right)\left(1-a q^{n}\right)\left(1-b q^{n}\right)\left(1-c q^{n}\right)\left(1-a b q^{n}\right)\left(1-a b q^{n} / c\right)}{\left(1-a b q^{2 n-1}\right)\left(1-a b q^{2 n}\right)\left(1-a b q^{2 n}\right)\left(1-a b q^{2 n+1}\right)}
$$

So

$$
\lambda_{1} \cdots \lambda_{j}=\frac{(-a c)^{j} q^{j(j+3) / 2}(q, a q, b q, c q, a b q, a b q / c ; q)_{j}}{\left(a b q, a b q^{2} ; q\right)_{2 j}} .
$$

Therefore

$$
\begin{aligned}
Q_{j}(t, a, b, c)= & \frac{1}{\lambda_{1} \cdots \lambda_{j}} \mathcal{L}\left(P_{j}(x) /(x t ; q)_{\infty}\right) \\
= & \frac{(-1)^{j}(1-q)^{j} q^{\left({ }_{2}{ }_{2}\right)}\left(a q^{j+1}, b q^{j+1}, c q^{j+1}, a b q^{j+1} / c ; q\right)_{\infty}}{(q ; q)_{j} a q(1-q)\left(q, a b q^{2}, c / a, a q / c ; q\right)_{\infty}} \\
& \quad \times \int_{c q}^{a q} \frac{\mathcal{D}_{q}^{j} w_{1}\left(x, a q^{j}, b q^{j}, c q^{j}\right)}{(x t ; q)_{\infty}} d_{q} x \\
= & \frac{t^{j} q^{-j}\left(a b q^{2} ; q\right)_{2 j}\left(a q^{j+1}, b q^{j+1}, c q^{j+1}, a b q^{j+1} / c ; q\right)_{\infty}}{a(q ; q)_{j}\left(q, a b q^{2}, c / a, a q / c ; q\right)_{\infty}} I_{j},
\end{aligned}
$$


where

$$
\begin{aligned}
& I_{j}=\frac{1}{q(1-q)} \int_{c q}^{a q} \frac{\left(x q^{-j} / a, x q^{-j} / c ; q\right)_{\infty}}{\left(x, b x / c, x t q^{-j} ; q\right)_{\infty}} d_{q} x \\
& =a \sum_{n=0}^{\infty} \frac{\left(q^{n+1}, a q^{n+1} / c ; q\right)_{\infty}}{\left(a q^{n+j+1}, a b q^{n+j+1} / c ; q\right)_{\infty}} \frac{q^{n+j}}{\left(a t q^{n+1} ; q\right)_{\infty}} \\
& -c \sum_{n=0}^{\infty} \frac{\left(q^{n+1}, c q^{n+1} / a ; q\right)_{\infty}}{\left(c q^{n+j+1}, b q^{n+j+1} ; q\right)_{\infty}} \frac{q^{n+j}}{\left(c t q^{n+1} ; q\right)_{\infty}} \\
& =\frac{a q^{j}(a q / c, q ; q)_{\infty}}{\left(a q^{j+1}, a b q^{j+1} / c, a t q ; q\right)_{\infty}} 3 \phi_{2}\left(\begin{array}{cc|c}
a q^{j+1}, & a b q^{j+1} / c, & a t q \\
a q / c, & 0 & q, q)
\end{array}\right. \\
& -\frac{c q^{j}(c q / a, q ; q)_{\infty}}{\left(c q^{j+1}, b q^{j+1}, c t q ; q\right)_{\infty}}{ }_{3} \phi_{2}\left(\begin{array}{cc|c}
c q^{j+1}, & b q^{j+1}, & c t q \\
c q / a, & 0
\end{array} \mid q, q\right) .
\end{aligned}
$$

The conclusion of the above calculations is that

$$
\begin{aligned}
& Q_{j}(t, a, b, c)=\frac{t^{j}}{(q ; q)_{j}\left(a b q^{2 j+2}, q\right)_{\infty}} \\
& \times\left[\frac { ( b q ^ { j + 1 } , c q ^ { j + 1 } ; q ) _ { \infty } } { ( c / a , a t q ; q ) _ { \infty } } \phi _ { 2 } \left(\begin{array}{cc|c}
a q^{j+1}, & a b q^{j+1} / c, & a t q \\
a q / c, & 0 & q, q)
\end{array}\right.\right.
\end{aligned}
$$

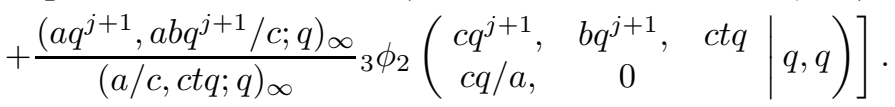

Next we apply (12.5.8) in [15] with $A=D /(q a t), B=a q^{j+1}, C=a b q^{j+1} / c, E=$ $a b q^{2 j+2}$ and let $D \rightarrow 0$ and realize that $Q_{j}(t, a, b, c)$ has the representation

$$
Q_{j}(t, a, b, c)=\frac{t^{j}}{(q ; q)_{j}(a q t, q)_{\infty}}{ }_{2} \phi_{1}\left(\begin{array}{c}
a q^{j+1}, a b q^{j+1} / c \\
a b q^{2 j+2}
\end{array} \mid q, q c t\right)
$$

It is clear from (4.35) that $Q_{j}(t, a, b, c)=\frac{t^{j}}{(q ; q)_{j}}+\cdots$.

Next we compute the functions $\widetilde{Q}_{j}(t, a, b, c)$. We have

$$
\begin{aligned}
\widetilde{Q}_{j}(t, a, b, c) & =\frac{1}{\lambda_{1} \cdots \lambda_{j}} \mathcal{L}\left(P_{j}(x)(-x t ; q)_{\infty}\right) \\
& =\frac{(-1)^{j} q^{\left(\begin{array}{l}
j \\
2
\end{array}\right)}(1-q)^{j}\left(a q^{j+1}, b q^{j+1}, c q^{j+1}, a b q^{j+1} / c ; q\right)_{\infty}}{a(q ; q)_{j}\left(q, a b q^{2+2 j}, c / a, a q / c ; q\right)_{\infty}} \\
& \times \int_{c q}^{a q}(-x t ; q)_{\infty} \mathcal{D}_{q}^{j} w_{1}\left(x, a q^{j}, b q^{j}, c q^{j}\right) d_{q} x \\
& =\frac{t^{j} q^{\left(\begin{array}{l}
j \\
2
\end{array}\right)}\left(a q^{j+1}, b q^{j+1}, c q^{j+1}, a b q^{j+1} / c ; q\right)_{\infty}}{a(q ; q)_{j}\left(q, a b q^{2+2 j}, c / a, a q / c ; q\right)_{\infty}} \tilde{I}_{j},
\end{aligned}
$$


where

$$
\begin{aligned}
\tilde{I}_{j}= & \frac{q^{-j}}{q(1-q)} \int_{c q}^{a q} \frac{\left(x q^{-j} / a, x q^{-j} / c ; q\right)_{\infty}}{\left(x, b x / c, x t q^{-j} ; q\right)_{\infty}} d_{q} x \\
= & a q^{-j} \sum_{n=0}^{\infty} \frac{\left(-a t q^{n+1}, q^{n+1-j}, a q^{n+1-j} / c ; q\right)_{\infty}}{\left(a q^{n+1}, a b q^{n+1} / c ; q\right)_{\infty}} q^{n} \\
& -c q^{-j} \sum_{n=0}^{\infty} \frac{\left(-c t q^{n+1}, q^{n+1-j}, c q^{n+1-j} / a ; q\right)_{\infty}}{\left(c q^{n+1}, b q^{n+1} ; q\right)_{\infty}} q^{n} \\
= & \frac{a\left(-a t q^{j+1}, q, a q / c ; q\right)_{\infty}}{\left(a q^{j+1}, a b q^{j+1} / c ; q\right)_{\infty}}{ }_{3} \phi_{2}\left(\begin{array}{cc|c}
a q^{j+1}, & a b q^{j+1} / c, & 0 \\
-a t q^{j+1}, & a q / c
\end{array} \mid q, q\right) \\
- & \frac{c\left(-c t q^{j+1}, q, c q / a ; q\right)_{\infty}}{\left(c q^{j+1}, b q^{j+1} ; q\right)_{\infty}}{ }_{3} \phi_{2}\left(\begin{array}{cc|c}
c q^{j+1}, & b q^{j+1}, & 0 \\
-c t q^{j+1}, & c q / a
\end{array} \mid q, q\right) .
\end{aligned}
$$

It follows that

$$
\begin{aligned}
& \widetilde{Q}_{j}(t, a, b, c)=\frac{t^{j} q^{\left(\begin{array}{c}
j \\
2
\end{array}\right)}}{(q ; q)_{j}\left(a b q^{2+2 j} ; q\right)_{\infty}} \\
& \times\left[\frac{\left(b q^{j+1}, c q^{j+1},-a t q^{j+1} ; q\right)_{\infty}}{(c / a ; q)_{\infty}}{ }_{3} \phi_{2}\left(\begin{array}{cc|c}
a q^{j+1}, & a b q^{j+1} / c, & 0 \\
-a t q^{j+1}, & a q / c & q, q
\end{array}\right)\right. \\
& \left.+\frac{\left(a q^{j+1}, a b q^{j+1} / c,-c t q^{j+1} ; q\right)_{\infty}}{(a / c ; q)_{\infty}} \phi_{2}\left(\begin{array}{cc|c}
c q^{j+1}, & b q^{j+1}, & 0 \\
-c t q^{j+1}, & c q / a
\end{array} \mid q, q\right)\right] .
\end{aligned}
$$

To simplify (4.36) we apply (12.5.8) in [15] with $B=a q^{j+1}, C=a b q^{j+1} / c, D=$ $-a t q^{j+1}, E=a b q^{2 j+2}$ and let $A \rightarrow \infty$. Alternately we may use the result in Exercise 3.8 of [12]. The conclusion is that

$$
\widetilde{Q}_{j}(t, a, b, c)=\frac{q^{\left(\begin{array}{c}
j \\
2
\end{array}\right) t^{j}\left(-a t q^{j+1} ; q\right)_{\infty}}}{(q ; q)_{j}} \phi_{2}\left(\begin{array}{cc|c}
a q^{j+1}, & a b q^{j+1} / c \\
a b q^{2 j+2}, & -a t q^{j+1} & q,-t c q^{j+1}
\end{array}\right)
$$

Finally the transformation (2.23) gives yet another alternate representation, namely

$$
\widetilde{Q}_{j}(t, a, b, c)=\frac{q^{\left(\begin{array}{c}
j \\
2
\end{array}\right)} t^{j}}{(q ; q)_{j}}(-t ; q)_{\infty 2} \phi_{1}\left(\begin{array}{c|c}
a q^{j+1}, c q^{j+1} \\
a b q^{2 j+2}
\end{array} \mid q,-t\right)
$$

It is clear from (4.37) or (4.38) that $\widetilde{Q}_{j}(t, a, b, c)=\frac{\left.t^{j} q^{(j} \begin{array}{l}(4) \\ 2\end{array}\right)}{(q ; q)_{j}}+\cdots$.

Theorem 4.3. The functions $Q_{j}(t, a, b, c)$ and $\widetilde{Q}_{j}(t, a, b, c)$ associated with the big q-Jacobi functional (4.30) are defined in (4.35) and (4.38) (or in (4.37)). Moreover we have the following addition formula:

$$
\begin{aligned}
& \frac{(-q a s ; q)_{\infty}}{(-s ; q)_{\infty}}{ }_{3} \phi_{2}\left(\begin{array}{ccc}
q a, & q a b / c & -s / t \\
a b q^{2}, & -q a s & q, q c t)
\end{array}\right. \\
& =\sum_{j=0}^{\infty} \frac{(-a c s t)^{j} q^{j(j+1)}(a q, b q, c q, a b q, a b q / c ; q)_{j}}{(q ; q)_{j}\left(a b q, a b q^{2} ; q\right)_{2 j}}
\end{aligned}
$$

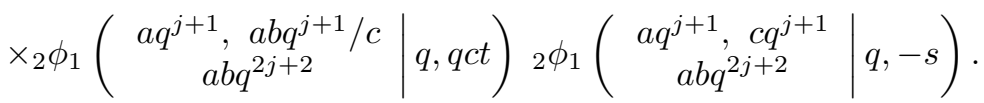


Proof. We only need to evaluate $\mathcal{T}_{s, q} Q_{0}(t ; a, b, c)$. Clearly $\mathcal{T}_{s, q} Q_{0}(t ; a, b, c)$ is

$$
\begin{aligned}
& \sum_{n=0}^{\infty} \frac{(q a, q a b / c ; q)_{n}}{\left(q, a b q^{2} ; q\right)_{n}}(q c)^{n} \sum_{k=0}^{\infty} \frac{(q a)^{k}}{(q ; q)_{k}} \mathcal{T}_{s, q} t^{n+k} \\
& =\sum_{n=0}^{\infty} \frac{(q a, q a b / c ; q)_{n}}{\left(q, a b q^{2} ; q\right)_{n}}(q c)^{n} \sum_{k=0}^{\infty} \frac{(q a)^{k}}{(q ; q)_{k}} t^{n+k}(-s / t ; q)_{n+k} \\
& =\sum_{n=0}^{\infty} \frac{(q a, q a b / c-s / t ; q)_{n}}{\left(q, a b q^{2} ; q\right)_{n}}(q c t)^{n}{ }_{1} \phi_{0}\left(-q^{n} s / t ;--; q, q a t\right) \\
& =\sum_{n=0}^{\infty} \frac{(q a, q a b / c-s / t ; q)_{n}}{\left(q, a b q^{2} ; q\right)_{n}}(q c t)^{n} \frac{\left(-q^{n+1} a s ; q\right)_{\infty}}{(a q t ; q)_{\infty}} .
\end{aligned}
$$

The $q$-binomial theorem reduces the last expression to

$$
\frac{(-q a s ; q)_{\infty}}{(q a t ; q)_{\infty}}{ }_{3} \phi_{2}\left(\begin{array}{ccc|}
q a, & q a b / c, & -s / t \\
a b q^{2}, & -q a s & q, q c t)
\end{array}\right.
$$

and the theorem follows.

4.4. Second $q$-addition formula. Define the noncommutative operation $\mathcal{S}_{y}$ on $f(x)=\sum_{n=0}^{\infty} a_{n} x^{n}$ by

$$
\mathcal{S}_{y} f(x)=\sum_{n=0}^{\infty} a_{n}(x+y)^{n},
$$

where $y x=q x y$. Recall [12, p.28] that the noncommutative binomial theorem reads

$$
(x+y)^{n}=\sum_{k=0}^{n}\left[\begin{array}{l}
n \\
k
\end{array}\right]_{q} x^{k} y^{n-k} .
$$

Theorem 4.4. The convolution identity (2.10) is equivalent to the noncommutative addition formula

$$
\mathcal{S}_{s} Q_{0}(t ; q)=\sum_{n=0}^{\infty} \lambda_{1} \cdots \lambda_{n} Q_{n}(t ; q) Q_{n}(s ; q) .
$$

Moreover, we have

$$
Q_{j}(t ; q)=\frac{1}{\lambda_{1} \cdots \lambda_{j}} \mathcal{L}\left(\frac{P_{j}(x)}{(x t ; q)_{\infty}}\right) .
$$

Proof. The noncommutative binomial theorem and the definition of $S_{y}$ give

$$
S_{y} x^{n}=\sum_{k=0}^{n}\left[\begin{array}{l}
n \\
k
\end{array}\right]_{q} x^{n-k} y^{k}
$$

Multiplying (2.10) by $t^{m} s^{n} /(q ; q)_{m}(q ; q)_{n}$ and summing over all $m, n \geq 0$, we get

$$
\sum_{m, n \geq 0} H_{0, m+n} \frac{t^{m} s^{n}}{(q ; q)_{m}(q ; q)_{n}}=\sum_{j, m, n \geq 0} \frac{t^{m} s^{n}}{(q ; q)_{m}(q ; q)_{n}} H_{j, m} H_{j, n} \lambda_{1} \ldots \lambda_{j} .
$$

This establishes the equivalence of (4.41) and (2.10). Equation (4.42) follows from Euler's formula. 
Example. We consider the Rogers-Szegö polynomials, which are defined by

$$
h_{n}(a ; q)=\sum_{k=0}^{n}\left[\begin{array}{l}
n \\
k
\end{array}\right]_{q} a^{k} .
$$

They have the $q$-exponential generating function

$$
Q_{0}(t ; q)=\sum_{n=0}^{\infty} \frac{h_{n}(a ; q)}{(q ; q)_{n}} t^{n}=\frac{1}{(t ; q)_{\infty}(a t ; q)_{\infty}} .
$$

Therefore

$$
\begin{aligned}
\mathcal{S}_{s} Q_{0}(t ; q) & =\sum_{n=0}^{\infty} \frac{h_{n}(a ; q)}{(q ; q)_{n}}(t+s)^{n} \\
& =\frac{1}{(t+s ; q)_{\infty}(a(t+s) ; q)_{\infty}} .
\end{aligned}
$$

The corresponding orthogonal polynomials are Al-Salam-Carlitz polynomials $U_{n}^{(a)}(x ; q)$, which have the generating function

$$
\frac{(t, a t ; q)_{\infty}}{(x t ; q)_{\infty}}=\sum_{n=0}^{\infty} \frac{U_{n}^{(a)}(x ; q)}{(q ; q)_{n}} t^{n}
$$

The associated functional is defined by

$$
\mathcal{L} f(x)=\frac{1}{(1-q)(q, a, q / a ; a)_{\infty}} \int_{a}^{1}(q x, q x / a ; q)_{\infty} f(x) d_{q} x .
$$

Note that, [18,

$$
\lambda_{j}=-a q^{j-1}\left(1-q^{j}\right) .
$$

It follows from (4.44) that the generating function of $Q_{n}(x)$ is

$$
\begin{aligned}
\sum_{n=0}^{\infty} & \lambda_{1} \cdots \lambda_{n} Q_{n}(t ; q) \frac{y^{n}}{(q ; q)_{n}} \\
& =\frac{(y, a y ; q)_{\infty}}{(1-q)(q, a, q / a ; q)_{\infty}} \int_{a}^{1} \frac{(q x, q x / a ; q)_{\infty}}{(x y, x t ; q)_{\infty}} d_{q} x \\
& =\frac{(a y ; q)_{\infty}}{(a, t ; q)_{\infty}}{ }_{2} \phi_{1}\left(\begin{array}{c}
y, \quad t \\
q / a
\end{array} \mid q, q\right)+\frac{(y ; q)_{\infty}}{(1 / a, a t ; q)_{\infty}}{ }_{2} \phi_{1}\left(\begin{array}{c}
a y, a t \\
a q
\end{array} \mid q, q\right) \\
& =\frac{(a y t, y q / t, q / a y t ; q)_{\infty}}{(t, a t, q / t, q / a t ; q)_{\infty}}{ }_{2} \phi_{1}\left(\begin{array}{c}
y, a y \\
y q / t
\end{array} \mid q, q / a y t\right),
\end{aligned}
$$

where the last equality follows from the transformation [12, (III.32)] if we replace all the small letters by capital ones and apply the parameter identification:

$$
A=y, \quad B=a y, \quad C=y q / t, \quad Z=q / a y t .
$$


Therefore

$$
\begin{aligned}
& \sum_{n=0}^{\infty} \lambda_{1} \cdots \lambda_{n} Q_{n}(t ; q) \frac{y^{n}}{(q ; q)_{n}}=\frac{(a y t, y q / t, q / a y t ; q)_{\infty}}{(t, a t, q / t, q / a t ; q)_{\infty}} \lim _{\delta \rightarrow 1^{-}}{ }_{2} \phi_{1}\left(\begin{array}{c}
y, a y \\
y q / t
\end{array} \mid q, q \delta / a y t\right) \\
& =\frac{(a y t, y q / t ; q)_{\infty}}{(t, a t, q / t, q / a t ; q)_{\infty}} \lim _{\delta \rightarrow 1^{-}}(\delta q ; q)_{\infty} \phi_{1}\left(\begin{array}{c}
q / t, q / a t \\
y q / t
\end{array} \mid q, \delta\right) \\
& =\frac{(a y t ; q)_{\infty}}{(t, a t ; q)_{\infty}}
\end{aligned}
$$

where we used the transformation [12, (III.3)] and $\lim _{\delta \rightarrow 1^{-}}(1-\delta) \sum_{n=0}^{\infty} a_{n} \delta^{n}=$ $\lim _{n \rightarrow \infty} a_{n}$. By equating the coefficients of $y^{n}$ we get

$$
Q_{n}(t ; q)=\frac{t^{n}}{(q ; q)_{n}} \frac{1}{(t, a t ; q)_{\infty}} .
$$

Summarizing we get the following theorem.

Theorem 4.5. If $s t=q t s$, then the following addition formula holds:

$$
\frac{1}{(t+s, a(t+s) ; q)_{\infty}}=\sum_{j=0}^{\infty}(-a)^{j} q^{\left(\begin{array}{c}
j \\
2
\end{array}\right)}(q ; q)_{j} \frac{t^{j}}{(q ; q)_{j}(t, a t ; q)_{\infty}} \frac{s^{j}}{(q ; q)_{j}(s, a s ; q)_{\infty}} .
$$

4.5. Computing first $q$-addition formulas using generating functions. Recall that the Al-Salam-Carlitz polynomials $\left\{U_{n}^{(a)}(x ; q)\right\}$ have the generating function (4.44) and the associated functional is in (4.45). It follows from (4.44) that the generating function of $Q_{n}(t ; q)$ is

$$
\begin{aligned}
& \sum_{n=0}^{\infty} \lambda_{1} \cdots \lambda_{n} Q_{n}(t ; q) \frac{y^{n}}{(q ; q)_{n}} \\
& \quad=\frac{(y, a y ; q)_{\infty}}{(1-q)(q, a, q / a ; q)_{\infty}} \int_{a}^{1} \frac{(q x, q x / a ; q)_{\infty}}{(x y, x t ; q)_{\infty}} d_{q} x \\
& \quad=\frac{(a y ; q)_{\infty}}{(a, t ; q)_{\infty}}{ }_{2} \phi_{1}\left(\begin{array}{c}
y, \quad t \\
q / a
\end{array} \mid q, q\right)+\frac{(y ; q)_{\infty}}{(1 / a, a t ; q)_{\infty}}{ }_{2} \phi_{1}\left(\begin{array}{c}
a y, \quad a t \\
a q
\end{array} \mid q, q\right) \\
& \quad=\frac{(a y t, y q / t, q / a y t ; q)_{\infty}}{(t, a t, q / t, q / a t ; q)_{\infty}}{ }_{2} \phi_{1}\left(\begin{array}{c}
y, a y \mid \\
y q / t
\end{array} \mid q, q / a y t\right),
\end{aligned}
$$

where the last equality follows from the transformation [12, (III.32)] if we replace all the small letters by capital ones and then take the following substitutions:

$$
A=y, \quad B=a y, \quad C=y q / t, \quad Z=q / a y t .
$$

Therefore

$$
\begin{aligned}
\sum_{n=0}^{\infty} \lambda_{1} \cdots \lambda_{n} Q_{n}(t ; q) \frac{y^{n}}{(q ; q)_{n}} & =\frac{(a y t, y q / t, q / a y t ; q)_{\infty}}{(t, a t, q / t, q / a t ; q)_{\infty}} \lim _{\delta \rightarrow 1}{ }_{2} \phi_{1}\left(\begin{array}{c}
y, \quad a y \\
y q / t
\end{array} \mid q, q \delta / a y t\right) \\
& =\frac{(a y t ; q)_{\infty}}{(t, a t ; q)_{\infty}} .
\end{aligned}
$$

Equating the coefficients of $y^{n}$ in the above identity we get

$$
Q_{n}(t ; q)=\frac{t^{n}}{(q ; q)_{n}} \frac{1}{(t, a t ; q)_{\infty}} .
$$


Similarly we have

$$
\sum_{n=0}^{\infty} \lambda_{1} \cdots \lambda_{n} \widetilde{Q}_{n}(t ; q) \frac{y^{n}}{(q ; q)_{n}}=\frac{(y, a y ; q)_{\infty}}{(1-q)(q, a, q / a ; q)_{\infty}} \int_{a}^{1} \frac{(q x, q x / a ; q)_{\infty}}{(x y ; q)_{\infty}}(-x t ; q)_{\infty} d_{q} x .
$$

The right-hand side of (4.50) is

$$
\begin{aligned}
& \frac{(y, a y ; q)_{\infty}}{(a, q / a ; q)_{\infty}}\left[\sum_{n=0}^{\infty} \frac{\left(q^{n+1} / a,-t q^{n} ; q\right)_{\infty}}{\left.(q ; q)_{n}\left(y q^{n} ; q\right)_{\infty}\right)} q^{n}-a \sum_{n=0}^{\infty} \frac{\left(a q^{n+1},-t a q^{n} ; q\right)_{\infty}}{(q ; q)_{n}\left(a y q^{n} ; q\right)_{\infty}} q^{n}\right] \\
= & \frac{(a y,-t ; q)_{\infty}}{(a ; q)_{\infty}}{ }_{3} \phi_{2}\left(\begin{array}{c}
y, 0,0 \\
-t, q / a
\end{array} \mid q, q\right)+\frac{(y,-a t ; q)_{\infty}}{(1 / a ; q)_{\infty}}{ }_{3} \phi_{2}\left(\begin{array}{c}
a y, 0,0 \\
q a,-a t
\end{array} \mid q, q\right) .
\end{aligned}
$$

In (III.34) of 12 replace $a, b, c, d, e$ by $A, B, C, D, E C$, respectively, then let $C \rightarrow 0$, and then let $A \rightarrow \infty$. The result is the three-term relation

$$
\begin{aligned}
{ }_{1} \phi_{1}\left(\begin{array}{c}
B \\
D
\end{array} \mid q,\right. & \left.\frac{D E}{B}\right)=\frac{(E ; q)_{\infty}}{(E / B ; q)_{\infty}}{ }_{3} \phi_{2}\left(\begin{array}{c}
B, 0,0 \\
D, q B / E
\end{array} \mid q, q\right) \\
& +\frac{(B, D E / B ; q)_{\infty}}{(D, B / E ; q)_{\infty}}{ }_{3} \phi_{2}\left(\begin{array}{c}
E, 0,0 \\
D E / B, q E / B
\end{array} \mid q, q\right) .
\end{aligned}
$$

We now choose $B=y, E=a y, D=-t$. Therefore (4.50) becomes

$$
\sum_{n=0}^{\infty} \lambda_{1} \cdots \lambda_{n} \widetilde{Q}_{n}(t ; q) \frac{y^{n}}{(q ; q)_{n}}=(-t ; q)_{\infty} \phi_{1}\left(\begin{array}{c}
y \\
-t
\end{array} \mid q,-a t\right) .
$$

Finally we apply the $q$-binomial theorem in the form

$$
(y ; q)_{n}=\sum_{k=0}^{n} \frac{(q ; q)_{n}}{(q ; q)_{k}(q ; q)_{n-k}} q^{\left(\begin{array}{c}
k \\
2
\end{array}\right)}(-y)^{k}
$$

and (4.46) to obtain

$$
\widetilde{Q}_{j}(t ; q)=\frac{\left(-t q^{j} ; q\right)_{\infty}}{(q ; q)_{j}} t^{j} q^{\left(\begin{array}{c}
j \\
2
\end{array}\right)}{ }_{1} \phi_{1}\left(0 ;-t q^{j} ; q,-a t q^{j}\right) .
$$

Theorem 4.6. We have the identity

$$
\begin{aligned}
& \frac{(-s ; q)_{\infty}}{(t ; q)_{\infty}}{ }_{2} \phi_{1}\left(\begin{array}{c}
0,-s / t \\
-s
\end{array} \mid q, a t\right) \\
= & \frac{1}{(t, a t ; q)_{\infty}} \sum_{n=0}^{\infty} \frac{\left(-s q^{n} ; q\right)_{\infty}}{(q ; q)_{n}}(-a s t)^{n} q^{n(n-1)}{ }_{1} \phi_{1}\left(0 ;-t q^{n} ; q,-a t q^{n}\right) .
\end{aligned}
$$

Proof. We only need to show that the left-hand side in (4.53) is $\mathcal{T}_{s, q} Q_{0}(t ; q)$. This can be seen as follows. By (4.43) and (4.8) we have

$$
\begin{aligned}
\mathcal{T}_{s, q} Q_{0}(t ; q) & =\sum_{n=0}^{\infty} \sum_{k=0}^{n} \frac{t^{n} a^{k}(-s / t ; q)_{n}}{(q ; q)_{k}(q ; q)_{n-k}} \\
& =\sum_{k=0}^{\infty} \frac{t^{k} a^{k}(-s / t ; q)_{k}}{(q ; q)_{k}} \sum_{n=0}^{\infty} \frac{t^{n}\left(-s q^{k} / t ; q\right)_{n}}{(q ; q)_{n}} \\
& =\frac{(-s ; q)_{\infty}}{(t ; q)_{\infty}}{ }_{2} \phi_{1}\left(\begin{array}{c}
0,-s / t \\
-s
\end{array} \mid q, a t\right) .
\end{aligned}
$$




\section{SHEFFER-TYPE POLYNOMIALS}

5.1. Moments of Sheffer-type polynomials. For the Sheffer-type orthogonal polynomials we can compute $Q_{j}(t)$ by the generating function

$$
\sum_{n=0}^{\infty} \lambda_{1} \ldots \lambda_{n} \alpha_{n} Q_{n}(t) \frac{y^{n}}{n !}=\mathcal{L}\left(e^{x t} \sum_{n=0}^{\infty} \alpha_{n} P_{n}(x) \frac{y^{n}}{n !}\right),
$$

where the $\alpha_{j}$ 's are some suitably chosen constants.

The cases of Hermite, Laguerre, Meixner, and Charlier polynomials do not lead to interesting addition theorems because the addition theorem predicted by Theorem 2.1 follows from the binomial theorem. We just indicate the corresponding $Q_{j}(t)$ in the addition formula of each family of polynomials.

Indeed in the case of Hermite polynomials $\left\{H_{n}(x)\right\}$,

$$
\lambda_{n}=n / 2, \quad H_{n}(x)=2^{n} P_{n}(x),
$$

and

$$
Q_{n}(t)=\frac{t^{n}}{n !} \exp \left(t^{2} / 4\right)
$$

In the case of Laguerre polynomials $\left\{L_{n}^{\alpha}(x)\right\}$,

$$
\lambda_{n}=n(\alpha+n), \quad L_{n}^{\alpha}(x)=\frac{(-1)^{n}}{n !} P_{n}(x),
$$

and

$$
Q_{n}(t)=\frac{t^{n}}{n !}(1-t)^{-\alpha-n-1} .
$$

For Meixner polynomials $\left\{M_{n}(x ; \beta, c)\right\}$, we have

$$
\lambda_{n}=\frac{n(n+\beta-1) c}{(1-c)^{2}}, \quad M_{n}(x ; \beta, c)=\frac{(c-1)^{n}}{c^{n}(\beta)_{n}} P_{n}(x),
$$

and

$$
Q_{n}(t)=\left(\frac{1-c}{1-c e^{t}}\right)^{\beta+n} \frac{\left(e^{t}-1\right)^{n}}{n !} .
$$

In the case of Charlier polynomials $\left\{C_{n}(x ; a)\right\}$,

$$
\lambda_{n}=a n, \quad C_{n}(x ; a)=(-a)^{-n} P_{n}(x) .
$$

A calculation gives

$$
Q_{j}(t)=\frac{\left(e^{t}-1\right)^{j}}{j !} \exp \left(e^{t}-1\right) .
$$

In the case of Meixner-Pollaczek polynomials $P_{n}^{\lambda}(x ; \phi)$, [18],

$$
\lambda_{n}=\frac{n(n+2 \lambda-1)}{4 \sin ^{2} \phi}, \quad P_{n}^{\lambda}(x ; \phi)=\frac{(2 \sin \phi)^{n}}{n !} P_{n}(x) .
$$

One can see that the $Q_{j}$ 's are given by

$$
Q_{j}(t)=\frac{2^{j}}{j !}\left(\frac{\sin \phi}{\sin (t / 2+\phi)}\right)^{2 \lambda+j}[\sin (t / 2)]^{j} .
$$

Note that for the orthogonal polynomials of Sheffer type all the $Q_{0}(t)$ 's have been given in [29]. 
5.2. Sheffer-type polynomials as moments. For the Hermite polynomials, we have

$$
\exp \left(2 x t-t^{2}\right)=\sum_{n=0}^{\infty} \frac{H_{n}(x)}{n !} t^{n}
$$

Let

$$
Q_{n}(t)=\frac{t^{n}}{n !} e^{2 x t-t^{2}}=\frac{t^{n}}{n !}+2(n+1) x \frac{t^{n+1}}{(n+1) !}+\cdots .
$$

From $\exp \left(2 x(t+s)-(t+s)^{2}\right)=\exp \left(2 x t-t^{2}+2 x s-s^{2}-2 t s\right)$ we derive the addition formula.

Theorem 5.1. The functions $\left\{Q_{j}(t)\right\}$ have the addition formula

$$
Q_{0}(t+s)=\sum_{n=0}^{\infty} n !(-2)^{n} Q_{n}(t) Q_{n}(s) .
$$

It follows that $\lambda_{n}=-2 n$ and

$$
H_{i, i+n}=\left(\begin{array}{c}
i+n \\
i
\end{array}\right) H_{n}(x) .
$$

Remark. Radoux 22 proved (5.13) by computing the corresponding Stieltjes tableau using induction.

For the Laguerre polynomials $L_{n}^{(\alpha)}(x)$ we have [18, p. 48]:

$$
e^{t}{ }_{0} F_{1}(-; \alpha+1 ;-x t)=\sum_{n=0}^{\infty} \frac{n ! L_{n}^{(\alpha)}(x)}{(\alpha+1)_{n}} \frac{t^{n}}{n !} .
$$

In (3.8), letting $B=\alpha+\beta+1$ and substituting $t$ and $s$ by $t / \alpha$ and $s / \alpha$, respectively, and then letting $\alpha \rightarrow \infty$ we get

$$
\begin{aligned}
{ }_{0} F_{1}(-; B+1 ;-2(t+s))=\sum_{n=0}^{\infty} & \frac{(-1)^{n}(B)_{n}(4 t s)^{n}}{(B)_{2 n}(B+1)_{n}}{ }_{0} F_{1}(-; B+2 n+1 ;-2 t) \\
& \times{ }_{0} F_{1}(-; B+2 n+1 ;-2 s) .
\end{aligned}
$$

Let

$$
Q_{n}(t ; \alpha)=\frac{t^{n}}{n !} e^{t}{ }_{0} F_{1}(-; \alpha+2 n+1 ;-2 x t), \quad n \geq 0 .
$$

Then we have the following addition formula.

Theorem 5.2. The functions $\left\{Q_{j}(t ; \alpha)\right\}$ have the addition formula

$$
Q_{0}(t+s ; \alpha)=\sum_{n=0}^{\infty} \frac{n !(\alpha)_{n}\left(-4 x^{2}\right)^{n}}{(\alpha)_{2 n}(\alpha+1)_{n}} Q_{n}(t ; \alpha) Q_{n}(s ; \alpha) .
$$

As an immediate consequence we have $\lambda_{n}=\frac{n(\alpha+n-1)\left(-4 x^{2}\right)}{(\alpha+2 n-1)(\alpha+2 n-2)(\alpha+n)}$ and

$$
H_{i, i+n}=\left(\begin{array}{c}
n+i \\
i
\end{array}\right) \frac{n ! L_{n}^{(\alpha+2 i)}(x)}{(\alpha+2 i+1)_{n}} .
$$

For the Meixner polynomials $M_{n}(x ; \beta, c)$ we have

$$
e^{t}{ }_{1} F_{1}\left(\begin{array}{c}
-x \\
\beta
\end{array} \mid\left(\frac{1-c}{c}\right) t\right)=\sum_{n=0}^{\infty} \frac{M_{n}(x ; \beta, c)}{n !} t^{n} .
$$


In (3.8), substituting $\alpha+1, \alpha+\beta+2, t$ and $s$ by $-x, \beta,(c-1) t /(2 c)$ and $(c-1) s /(2 c)$, respectively, we obtain

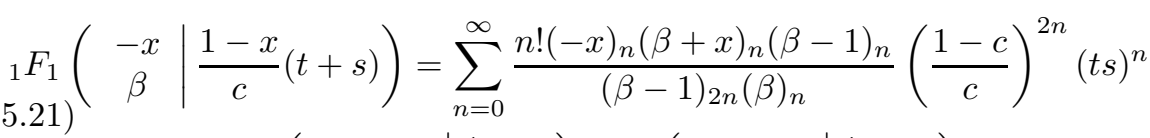

$$
\begin{aligned}
& \times{ }_{1} F_{1}\left(\begin{array}{c|c}
n-x \\
\beta+2 n
\end{array} \mid \frac{1-c}{c} t\right){ }_{1} F_{1}\left(\begin{array}{c|c}
n-x & 1-c \\
\beta+2 n & \frac{1-2 n}{c}
\end{array}\right) .
\end{aligned}
$$

Therefore, defining

$$
Q_{n}(x ; \beta, c)=\frac{t^{n}}{n !} e^{t} F_{1}\left(\begin{array}{c|c}
n-x & 1-c \\
\beta+2 n & \frac{1-}{c} t
\end{array}\right),
$$

we have the following addition formula.

Theorem 5.3. The functions $\left\{Q_{j}(t ; \beta, c)\right\}$ have the addition formula

$$
Q_{0}(t+s ; \beta, c)=\sum_{n=0}^{\infty} \frac{n !(-x)_{n}(\beta+x)_{n}(\beta-1)_{n}}{(\beta-1)_{2 n}(\beta)_{n}}\left(\frac{1-c}{c}\right)^{2 n} Q_{n}(t ; \beta, c) Q_{n}(s ; \beta, c) .
$$

In the same way we derive

$$
\lambda_{n}=\frac{n(-x+n-1)(\beta+x+n-1)(\beta+n-2)}{(\beta+2 n-2)(\beta+2 n-3)(\beta+n-1}\left(\frac{1-c}{c}\right)^{2}
$$

and

$$
H_{i, i+n}=\left(\begin{array}{c}
i+n \\
i
\end{array}\right) M_{n}(x-i ; \beta+2 i, c) .
$$

The Meixner-Pollaczek polynomials $P_{n}^{(\lambda)}(x ; \phi)$ have the generating function

$$
e^{t}{ }_{1} F_{1}\left(\begin{array}{c}
\lambda+i x \\
2 \lambda
\end{array} \mid\left(e^{-2 i \phi}-1\right) t\right)=\sum_{n=0}^{\infty} \frac{P_{n}^{(\lambda)}(x ; \phi)}{(2 \lambda)_{n} e^{i n \phi}} t^{n} .
$$

In (3.8), substituting $\alpha+1, \beta+1, t$ and $s$ by $\lambda-1+i x, \lambda-1-i x,\left(1-e^{-2 i \phi}\right) t / 2$ and $\left(1-e^{-2 i \phi}\right) s / 2$, respectively, and letting

$$
Q_{n}^{(\lambda)}(x ; \phi)=\frac{t^{n}}{n !} e^{t}{ }_{1} F_{1}\left(\begin{array}{c}
\lambda+i x+n \\
2 \lambda+2 n
\end{array} \mid\left(e^{-2 i \phi}-1\right) t\right),
$$

we obtain the following addition formula corresponding to Meixner-Pollaczek polynomials.

Theorem 5.4. We have the addition formula

$$
Q_{0}^{(\lambda)}(t+s ; \phi)=\sum_{n=0}^{\infty} \frac{n !(\lambda+i x)_{n}(\lambda-i x)_{n}(2 \lambda-1)_{n}}{(2 \lambda-1)_{2 n}(2 \lambda)_{n}} 4^{n} Q_{n}^{(\lambda)}(t ; \phi) Q_{n}^{(\lambda)}(s ; \phi)
$$

\section{6. $q$-UltrasphericAl AND ASKeY-Wilson POLYNOMIALS}

The continuous $q$-ultraspherical polynomials have the weight function

$$
w(x ; \beta)=\frac{1}{2 \pi} \frac{\left(e^{2 i \theta}, e^{-2 i \theta}\right)}{\left(\beta e^{2 i \theta}, \beta e^{-2 i \theta}\right)} \frac{\left(\beta^{2}, q\right)_{\infty}}{(\beta, \beta q)_{\infty}} \frac{1}{\sqrt{1-x^{2}}}, \quad x=\cos \theta \in(-1,1),
$$


and have the property

$$
C_{n}(x, \beta \mid q)=\frac{2^{n}(\beta)_{n}}{(q)_{n}} P_{n}(x), \quad \lambda_{j}=\frac{\left(1-q^{j}\right)\left(1-\beta^{2} q^{j-1}\right)}{4\left(1-\beta q^{j-1}\right)\left(1-\beta q^{j}\right)} .
$$

In view of (1.10) and (1.8) we see that

$$
e^{x y}=\frac{2}{y} \sum_{n=0}^{\infty}(n+1) I_{n+1}(y) U_{n}(x) .
$$

Now the special case $\gamma=q$ of the connection relation (1.9) gives the following expansion:

$$
U_{n}(x)=\sum_{k=0}^{[n / 2]} \frac{\beta^{k}(q / \beta ; q)_{k}(q ; q)_{n-k}}{(q ; q)_{k}(q \beta ; q)_{n-k}} \frac{1-\beta q^{n-2 k}}{1-\beta} C_{n-2 k}(x ; \beta \mid q) .
$$

Using the above expansion and the orthogonality of the $q$-ultraspherical polynomials we have

$$
\begin{aligned}
Q_{j}(t) & =\frac{2}{t} \frac{1}{\lambda_{1} \cdots \lambda_{j}} \sum_{n=0}^{\infty} \int_{-1}^{1} w(x)(n+1) I_{n+1}(t) U_{n}(x) P_{j}(x) d x \\
& =\frac{2^{j+1}(\beta)_{j}}{(q)_{j}} \frac{1}{t} \sum_{k=0}^{\infty}(j+2 k+1) I_{j+2 k+1}(t) \frac{\beta^{k}(q / \beta ; q)_{k}(q ; q)_{j+k}}{(q ; q)_{k}(q \beta ; q)_{j+k}} \frac{1-\beta q^{j}}{1-\beta} \\
& =\frac{2^{j+1}}{t} \sum_{k=0}^{\infty}(j+2 k+1) I_{j+2 k+1}(t) \frac{\beta^{k}(q / \beta ; q)_{k}\left(q^{j+1} ; q\right)_{k}}{(q ; q)_{k}\left(\beta q^{j+1} ; q\right)_{k}} .
\end{aligned}
$$

To denote the explicit dependence on $q$ and $\beta$ we set

$$
Q_{j}(t ; \beta, q)=\frac{2^{j+1}}{t} \sum_{k=0}^{\infty}(j+2 k+1) I_{j+2 k+1}(t) \frac{\beta^{k}(q / \beta ; q)_{k}\left(q^{j+1} ; q\right)_{k}}{(q ; q)_{k}\left(\beta q^{j+1} ; q\right)_{k}}
$$

Thus we have proved that

Theorem 6.1. The functions $\left\{Q_{j}(t ; \beta, q)\right\}$ have the addition formula

$$
Q_{0}(s+t ; \beta, q)=\sum_{n=0}^{\infty} \frac{(q ; q)_{n}\left(\beta^{2} ; q\right)_{n}}{4^{n}(\beta ; q)_{n}(q \beta ; q)_{n}} Q_{n}(s ; \beta, q) Q_{n}(t ; \beta, q) .
$$

The special case $\beta \rightarrow 0$ is worth recording. Indeed if

$$
F_{n}(t ; q):=\frac{2^{n+1}}{t} \sum_{k=0}^{\infty}(n+2 k+1) I_{n+2 k+1}(t)(-1)^{n} q^{\left(\begin{array}{c}
k+1 \\
2
\end{array}\right)}\left[\begin{array}{c}
n+k \\
k
\end{array}\right]_{q},
$$

then we have established the curious result

$$
F_{0}(s+t ; q)=\sum_{n=0}^{\infty} \frac{(q ; q)_{n}}{4^{n}} F_{n}(s ; q) F_{n}(t ; q) .
$$

Another interesting case is to let $\beta=q^{\nu}$; then let $q \rightarrow 1$. This should reduce (6.3) to (3.1) since $\lim _{q \rightarrow 1} C_{n}\left(x ; q^{\nu} \mid q\right)=C_{n}^{\nu}(x)$. Surprisingly the $q \rightarrow 1$ limit of (6.3), after setting $\beta=q^{\nu}$ is

$$
Q_{j}(t)=2^{j} \sum_{k=0}^{\infty} I_{j+2 k}(t) \frac{(-\nu)_{k}(j)_{k}}{k !(j+1+\nu)_{k}} \frac{j+2 k}{j} .
$$


Equating the above limit and the $Q_{j}$ as in (3.1) leads to the following known identity involving Bessel functions:

$$
(z / 2)^{\mu-\nu} J_{\nu}(z)=\sum_{n=0}^{\infty} \frac{\Gamma(\mu+n) \Gamma(\nu+1-\mu)(\mu+2 n)}{n ! \Gamma(\mu+1-\mu-n) \Gamma(\nu+n+1)} J_{\mu+2 n}(z) ;
$$

see [7, (7.15.2)]. It is also worth mentioning that (6.7) is equivalent to a theorem of Bailey evaluating the sum of a well-poised ${ }_{4} F_{3}$ with argument $-1,[6$, (4.5.4)] .

Next we consider the Askey-Wilson polynomials whose weight function is

$$
\begin{gathered}
W\left(x ; a_{1}, a_{2}, a_{3}, a_{4} \mid q\right)=\frac{\left(e^{2 i \theta}, e^{-2 i \theta} ; q\right)_{\infty}}{\prod_{j=1}^{4}\left(a_{j} e^{i \theta}, a_{j} e^{-i \theta} ; q\right)_{\infty}} \frac{1}{\sqrt{1-x^{2}}} \\
\times \frac{(q ; q)_{\infty} \prod_{1 \leq j<k \leq 4}\left(a_{j} a_{k} ; q\right)_{\infty}}{2 \pi\left(a_{1} a_{2} a_{3} a_{4} ; q\right)_{\infty}}, \quad x=\cos \theta .
\end{gathered}
$$

The Askey-Wilson polynomials have the basic hypergeometric function representation

$$
\begin{aligned}
p_{n}\left(x ; a_{1}, a_{2}, a_{3}, a_{4} \mid q\right)= & a_{1}^{-n}\left(a_{1} a_{2}, a_{1} a_{3}, a_{1} a_{4} ; q\right)_{n} \\
& \times{ }_{4} \phi_{3}\left(\begin{array}{c}
q^{-n}, a_{1} a_{2} a_{3} a_{4} q^{n-1}, a_{1} e^{i \theta}, a_{1} e^{-i \theta} \\
a_{1} a_{2}, a_{1} a_{3}, a_{1} a_{4}
\end{array} \mid q, q\right) .
\end{aligned}
$$

One very special case of their connection coefficients formula is [2, (6.4)-(6.5)]

$$
p_{n}\left(x ; \alpha, a_{2}, a_{3}, a_{4} \mid q\right)=\sum_{k=0}^{n} c_{k, n} p_{n}\left(x ; a, a_{2}, a_{3}, a_{4} \mid q\right),
$$

where

$$
c_{k, n}=\frac{a^{n-k}(q ; q)_{n}\left(\alpha a_{2} a_{3} a_{4} q^{n-1} ; q\right)_{k}(\alpha / a ; q)_{n-k}}{\left(q, a a_{2} a_{3} a_{4} q^{k-1} ; q\right)_{k}\left(q, a a_{2} a_{3} a_{4} q^{2 k} ; q\right)_{n-k}} \prod_{2 \leq j<m \leq 4}\left(a_{j} a_{m} q^{k} ; q\right)_{n-k} .
$$

Moreover

$$
\begin{gathered}
U_{n}(x)=\frac{1}{\left(q^{n+2} ; q\right)_{n}} p_{n}(x ; \sqrt{q}, q,-\sqrt{q},-q \mid q), \\
p_{n}(x ; a, b, c, d \mid q)=2^{n}\left(a b c d q^{n-1} ; q\right)_{n} P_{n}(x), \\
\lambda_{n}=\frac{\left(1-q^{n}\right)\left(1-a_{1} a_{2} a_{3} a_{4} q^{n-2}\right) \prod_{1 \leq j<k \leq 4}\left(1-a_{j} a_{k} q^{n-1}\right)}{4\left(a_{1} a_{2} a_{3} a_{4} q^{2 n-3}, a_{1} a_{2} a_{3} a_{4} q^{2 n-2} ; q\right)_{2}} .
\end{gathered}
$$

Applying (2.18) and the plane wave expansion (6.1) we find that the $Q_{m}$ 's are given by

$$
\begin{gathered}
Q_{m}(t)=\frac{1}{\lambda_{1} \lambda_{2} \cdots \lambda_{m}} \mathcal{L}\left(e^{x t} P_{m}\right)=\frac{2 / t}{\lambda_{1} \lambda_{2} \cdots \lambda_{m}} \sum_{n=0}^{\infty}(n+1) I_{n+1}(t) \mathcal{L}\left(U_{n}(x) P_{m}\right) \\
=(2 / t) \sum_{n=m}^{\infty}(n+1) I_{n+1}(t) \frac{\left(a q^{m+1} ; q\right)_{m}}{\left(q^{n+2} ; q\right)_{n}} 2^{n} c_{m, n} \\
=(2 / t) \sum_{n=m}^{\infty} 2^{n}(n+1) I_{n+1}(t) \frac{\left(a q^{m+1} ; q\right)_{m}}{\left(q^{n+2} ; q\right)_{n}} \frac{a^{n-m}(q ; q)_{n}\left(q^{n+2} ; q\right)_{m}(q / a ; q)_{n-m}}{\left(q, a q^{m+1} ; q\right)_{m}\left(q, a q^{2 m+2} ; q\right)_{n-m}} \\
\times\left(-q^{m+1}, q^{m+3 / 2},-q^{m+3 / 2} ; q\right)_{n-m} .
\end{gathered}
$$


After some simplification we arrive at

$$
\begin{aligned}
& Q_{m}(t)=\frac{2^{m+1}}{t} \sum_{n=0}^{\infty} 2^{n} a^{n}(n+m+1) \\
& \quad \times \frac{\left(q^{m+1}, q / a,-q^{m+1} ; q\right)_{n}\left(q^{2 m+3} ; q^{2}\right)_{n}}{\left(q, a q^{2 m+2}, q^{n+m+2} ; q\right)_{n}} I_{n+m+1}(t) .
\end{aligned}
$$

Theorem 6.2. The functions $\left\{Q_{m}(x)\right\}$ defined in (6.12) satisfy the addition theorem

$$
Q_{0}(s+t)=\sum_{n=0}^{\infty} \frac{\left(q^{2}, a^{2} q ; q\right)_{n}}{4^{n}\left(a q, a q^{2} ; q\right)_{2 n}} Q_{n}(t) Q_{n}(s)
$$

\section{Ultraspherical POLYNOMIALS AS MOMENTS}

One of the generating functions reads

$$
Q(t)=\sum_{n=0}^{\infty} \frac{C_{n}^{(\nu)}(x)}{(2 \nu)_{n}} t^{n}=e^{x t}{ }_{0} F_{1}\left(\begin{array}{c}
- \\
\nu+\frac{1}{2}
\end{array} ; \frac{\left(x^{2}-1\right) t^{2}}{4}\right) .
$$

Let $\cos \phi=-1$. Then $w=z+Z$ and $C_{n}^{\nu}(-1)=(-1)^{n} \frac{(2 \nu)_{n}}{n !}$. It follows from (1.5) that

$$
\begin{aligned}
& { }_{0} F_{1}\left(\begin{array}{c}
- \\
\nu+1 / 2
\end{array} ; \frac{-(z+Z)^{2}}{4}\right)=\sum_{n=0}^{\infty} \frac{(n+\nu-1 / 2)(-1)^{n}(2 \nu-1)_{n}}{n !(\nu+1 / 2)_{n}(\nu-1 / 2)_{n+1}} \\
& \times\left(\frac{z Z}{4}\right)^{n}{ }_{0} F_{1}\left(\begin{array}{c}
- \\
\nu+1 / 2+n
\end{array} ; \frac{-z^{2}}{4}\right){ }_{0} F_{1}\left(\begin{array}{c}
- \\
\nu+1 / 2+n
\end{array} ; \frac{-Z^{2}}{4}\right) .
\end{aligned}
$$

Therefore, letting $z=t \sqrt{1-x^{2}}$ and $Z=t \sqrt{1-x^{2}}$ we obtain

$$
Q(t+s)=\sum_{n=0}^{\infty} \frac{(n+\nu-1 / 2)(-1)^{n}(2 \nu-1)_{n}}{(\nu+1 / 2)_{n}(\nu-1 / 2)_{n+1}} \frac{\left(1-x^{2}\right)^{n} n !}{4^{n}} Q_{n}(t) Q_{n}(s),
$$

where

$$
Q_{n}(t)=\frac{t^{n}}{n !} e^{t x} F_{1}\left(\begin{array}{c}
- \\
\nu+1 / 2+n
\end{array} ; \frac{\left(x^{2}-1\right) t^{2}}{4}\right)=\frac{t^{n}}{n !}+(n+1) x \frac{t^{n+1}}{(n+1) !}+\cdots .
$$

Extracting the coefficients of $t^{m} s^{n}$ in (7.3) we get

$$
\begin{gathered}
\frac{(m+n) ! C_{m+n}^{\nu}(x)}{(2 \nu)_{m+n} m ! n !}=\sum_{k=0}^{\infty} \frac{(k+\nu-1 / 2)(-1)^{k}(2 \nu-1)_{k}}{k !(\nu+1 / 2)_{k}(\nu-1 / 2)_{k+1}} \frac{\left(1-x^{2}\right)^{k}}{4^{k}} \\
\times \frac{C_{m-k}^{\nu+k}(x) C_{n-k}^{\nu+k}(x)}{(2 \nu)_{m-k}(2 \nu)_{n-k}}
\end{gathered}
$$

Using the relation

$$
\lim _{\alpha \mapsto \infty} \alpha^{-n / 2} C_{n}^{\alpha+1 / 2}(x / \sqrt{\alpha})=\frac{H_{n}(x)}{n !},
$$

we derive

$$
\frac{H_{m+n}(x)}{m ! n !}=\sum_{k=0}^{m \wedge n} \frac{(-2)^{k}}{k !} \frac{H_{m-k}(x)}{(m-k) !} \frac{H_{n-k}(x)}{(n-k) !} .
$$

An immediate consequence of (17.3) is the following formula for the Hankel determinant evaluation [28, Corollary 3]. 
Corollary 7.1. We have

$$
\operatorname{det}\left(\frac{(i+j) !}{(2 \nu)_{i+j}} C_{i+j}^{\nu}(x)\right)_{0 \leq i, j \leq n}=\frac{\left(x^{2}-1\right)^{n(n+1) / 2}}{2^{n^{2}}} \prod_{r=1}^{n} \frac{r !(2 \nu)_{r-1}}{(\nu+1 / 2)_{r-1}(\nu+1 / 2)_{r}},
$$

and more generally, for $n \geq 0$, the entries of the Stieltjes tableau (2.9) are

$$
H_{i, i+n}=\sum_{k=0}^{\lfloor n / 2\rfloor} \frac{(n+i) ! x^{n-2 k}\left(x^{2}-1\right)^{k} t^{2 k}}{i ! k !(n-2 k) !(\nu+1 / 2+i)_{k} 4^{k}} .
$$

\section{A variation of the Stieltues-Rogers addition formula}

Let $\left\{P_{n}(x)\right\}$ satisfy (2.1) with moment sequence $\left\{\mu_{n}\right\}$ and $\bar{P}_{n}(x)=a^{-n} P_{n}(a x+b)$ $(a \neq 0)$. Then it is well known [5, p.25] that $\left\{\bar{P}_{n}(x)\right\}$ is an OPS with respect to the moments given by

$$
\bar{\mu}_{n}=a^{-n} \sum_{k=0}^{n}\left(\begin{array}{l}
n \\
k
\end{array}\right)(-b)^{n-k} \mu_{k}
$$

and satisfies

$$
\bar{P}_{n+1}(x)=\left(x-\frac{b_{n}-b}{a}\right) \bar{P}_{n}(x)-\frac{\lambda_{n}}{a^{2}} \bar{P}_{n-1}(x) .
$$

Let $Q_{0}(t)=\sum_{n=0}^{\infty} \mu_{n} \frac{t^{n}}{n !}$. Then it is easy to see that

$$
\bar{Q}_{0}(t)=\sum_{n=0}^{\infty} \bar{\mu}_{n} \frac{t^{n}}{n !}=e^{-b t / a} Q_{0}(t / a) .
$$

The following variation of the Stieltjes-Rogers addition formula (2.12) is sometimes very useful.

Theorem 8.1. The addition formula for the moment sequence (8.1) is

$$
\bar{Q}_{0}(s+t)=\sum_{n=0}^{\infty} \lambda_{1} \ldots \lambda_{n} a^{-2 n} \bar{Q}_{n}(s) \bar{Q}_{n}(t),
$$

where $\bar{Q}_{n}(t)=e^{-b t / a} Q_{n}(t / a)$. The corresponding entries in (2.9) are

$$
\bar{H}_{j, j+n}=\sum_{k=0}^{n+j}\left(\begin{array}{c}
n+j \\
k
\end{array}\right)(-b)^{k} a^{-n-j} H_{j, n+j-k} .
$$

In particular we have $\bar{H}_{n, n}=(-b / a)^{n} H_{n, n}$, i.e.,

$$
\operatorname{det}\left(\bar{\mu}_{i+j}\right)_{0 \leq i, j \leq n}=\left(\frac{-b}{a}\right)^{n} \operatorname{det}\left(\mu_{i+j}\right)_{0 \leq i, j \leq n} .
$$

For example, let $\mu_{n}=(\alpha+1)_{n}$ be the $n$ th-moment of the Laguerre polynomials $\left\{L_{n}^{\alpha}(x)\right\}$ (see (5.4)). If $a=b=1 / x$, then

$$
\bar{\mu}_{n}=\sum_{k=0}^{n}(-1)^{n-k}\left(\begin{array}{l}
n \\
k
\end{array}\right) x^{k}(\alpha+1)_{k} .
$$


is a weighted derangement number. The corresponding addition formula reads

$$
\bar{Q}_{0}(s+t)=\sum_{n=0}^{\infty} n !(\alpha+1)_{n} x^{2 n} \bar{Q}_{n}(s) \bar{Q}_{n}(t) .
$$

The $\alpha=0$ case of the above formula was derived by Radoux 22 using induction.

\section{ACKNOWLEDGMENTS}

We are grateful to Mizan Rahman for his simplification of the $Q_{j}$ functions of Sections 4 and 5 and to Tom Koornwinder for pointing out the work of Burchnall and Chaundy [4. We thank the referee for his careful reading and helpful remarks. This work was done during the visit of the first author to Université Lyon 1 and he gratefully acknowledges the hospitality and financial support of the Institute Camille Jordan.

\section{REFERENCES}

[1] G. E. Andrews, R. A. Askey, and R. Roy, Special Functions, Cambridge University Press, Cambridge, 1999. MR.1688958 (2000g:33001)

[2] R. Askey and J. Wilson, Some basic hypergeometric orthogonal polynomials that generalize Jacobi polynomials. Mem. Amer. Math. Soc. 54, Number 319 (1985), 55 pages. MR783216 (87a:05023)

[3] G. E. Andrews and J. Wimp, Some $q$-orthogonal polynomials and related Hankel determinants, Rocky Mountain J. Math., 32(2002), Number 2, 429-442. MR1934898 (2003i:33020)

[4] J. L. Burchnall and T. W. Chaundy, Expansions of Appell's double hypergeometric functions, II, Quarterly J. Math. 12 (1941), 112-128. MR0005208 (3:118b)

[5] T. S. Chihara, An introduction to orthogonal polynomials, Gordon and Breach, New York, 1978. MR0481884(58:1979)

[6] A. Erdélyi, W. Magnus, F. Oberhettinger, and F. G. Tricomi, Higher Transcendental Functions, volume 1, McGraw-Hill, New York, 1953. MR0058756 (15:419i)

[7] A. Erdélyi, W. Magnus, F. Oberhettinger, and F. G. Tricomi, Higher Transcendental Functions, volume 2, McGraw-Hill, New York, 1953. MR.0058756 (15:419i)

[8] J. L. Fields and M. E. H. Ismail, Polynomial expansions, Math. Comp. 29 (1975), 894-902. MR0372472 (51:8680)

[9] J. L. Fields and J. Wimp, Expansions of hypergeometric functions in hypergeometric functions, Math. Comp. 15 (1961), 390-395. MR0125992 (23:A3289)

[10] Ph. Flajolet, Combinatorial aspects of continued fractions, Discrete Math., 32 (1980), 125161. MR592851 (82f:05002a)

[11] A. R. Forsyth, Theory of Functions of a Complex Variable, 3rd edition, volumes 1 and 2, Cambridge University Press, 1918, reprinted by Dover Publications, New York, 1965. MR0178116 (31:2374)

[12] G. Gasper and M. Rahman, Basic Hypergeometric Series, second edition Cambridge University Press, Cambridge, 2004. MR.2128719 (2006d:33028)

[13] Q. H. Hou, A. Lascoux and Y. P. Mu, Continued fractions for Rogers-Szegő polynomials, Numerical Algorithms 35(2004), 81-90. MR2041804(2005d:33026)

[14] M. E. H. Ismail, Determinants with orthogonal polynomial entries, J. Comput. Appl. Math. 178 (2005), 255-266. MR2127884(2006h:33007)

[15] M. E. H. Ismail, Classical and Quantum Orthogonal Polynomials in one Variable, Cambridge University Press, Cambridge, 2005. MR2191786 (2007f:33001)

[16] M. E. H. Ismail and D. Stanton, More orthogonal polynomials as moments, B. Sagan, \& R. Stanley (Eds.), in "Mathematical Essays in Honor of Gian-Carlo Rota", Birkhäuser, Basel, 1998, pp. 377-396. MR.1627382 (99f:33011)

[17] F. H. Jackson, Basic double hypergeometric functions (II), Quart. J. Math., Oxford Ser. 15 (1944), pp. 49-61. MR0011348 (6:152e)

[18] R. Koekoek and R. Swarttouw, The Askey-scheme of hypergeometric orthogonal polynomials and its q-analogues, Reports of the Faculty of Technical Mathematics and Informatics no. 98-17, Delft University of Technology, Delft, 1998. 
[19] T.H. Koornwinder, The addition formula for Laguerre polynomials, SIAM J. Math. Anal. 8 (1977), 535-540. MR0454106 (56:12357)

[20] Ch. Krattenthaler, Advanced determinant calculus, Séminaire Lotharingien Combin. 42 ("The Andrews Festschrift") (1999), Article B42q, 67 pp. MR1701596 (2002i:05013)

[21] Ch. Krattenthaler, Advanced determinant calculus: A complement, Linear Algebra Appl. 411 (2005), 68-166. MR2178686 (2006g:05022)

[22] C. Radoux, Addition formulas for polynomials built on classical combinatorial sequences, J. Computational and Appl. Math. 115 (2000), 471-477. MR1747239 (2000m:05010)

[23] H. S. Wall, Analytic Theory of Continued Fractions, Van Nostrand, Princeton, NJ, 1948. MR0025596 (10:32d)

[24] A. Verma, Some transformations of series with arbitrary terms, Ist. Lombardo Accad. Sci. Lett. Rend. A 106 (1972), 342-353. MR0328144 (48:6486)

[25] X. G. Viennot, Une théorie combinatoire des polynômes orthogonaux généraux, Lecture Notes, 1983, Université du Québec à Montréal, Montreal.

[26] G. N. Watson, A Treatise on the Theory of Bessel Functions, second edition, Cambridge University Press, Cambridge, 1944. MR0010746 (6:64a)

[27] E. T. Whittaker and G. N. Watson, A Course of Modern Analysis, fourth edition, Cambridge University Press, Cambridge, 1927. MR1424469 (97k:01072)

[28] J. Wimp, Hankel determinants of some polynomials arising in combinatorial analysis, Numerical Algorithms, 24 (2000), 179-193. MR1784998 (2001g:15009)

[29] J. Zeng, Weighted derangements and the linearization coefficients of orthogonal Sheffer polynomials, Proc. London Math. Soc., t. 65, 1992, 1-22. MR.1162485 (93c:05003)

Department of Mathematics, University of Central Florida, Orlando, Florida 32816

Université de Lyon, Université Lyon 1, Institute Camille Jordan, UMR 5028 du CNRS, 69622 Villeurbanne, France 